University of Nebraska - Lincoln

DigitalCommons@University of Nebraska - Lincoln

\title{
The kinetic cluster-field method and its application to studies of L12-type orderings in alloys
}

\author{
Kirill D. Belashchenko \\ 'Kurchatov Institute' Russian Research Centre, belashchenko@unl.edu \\ V Yu Dobretsov \\ 'Kurchatov Institute' Russian Research Centre, dvy@relcom.ru \\ I R Pankratov \\ 'Kurchatov Institute' Russian Research Centre \\ G D Samolyuk \\ 'Kurchatov Institute' Russian Research Centre \\ V G Vaks \\ 'Kurchatov Institute' Russian Research Centre, vaks@mbslab.kiae.ru
}

Follow this and additional works at: https://digitalcommons.unl.edu/physicsbelashchenko

\footnotetext{
Belashchenko, Kirill D.; Dobretsov, V Yu; Pankratov, I R; Samolyuk, G D; and Vaks, V G, "The kinetic clusterfield method and its application to studies of L12-type orderings in alloys" (1999). Kirill Belashchenko Publications. 5.

https://digitalcommons.unl.edu/physicsbelashchenko/5
}

This Article is brought to you for free and open access by the Research Papers in Physics and Astronomy at DigitalCommons@University of Nebraska - Lincoln. It has been accepted for inclusion in Kirill Belashchenko Publications by an authorized administrator of DigitalCommons@University of Nebraska - Lincoln. 


\title{
The kinetic cluster-field method and its application to studies of $\mathrm{L1}_{2}$-type orderings in alloys
}

\author{
K D Belashchenko, V Yu Dobretsov, I R Pankratov, G D Samolyuk and \\ V G Vaks \\ 'Kurchatov Institute' Russian Research Centre, Moscow 123182, Russia
}

Received 21 July 1999, in final form 16 September 1999

\begin{abstract}
The earlier-described master equation approach to configurational kinetics of nonequilibrium alloys is applied to study $\mathrm{L}_{2}$-type orderings in FCC alloys. We describe the kinetic tetrahedron cluster-field method which generalizes a similar method used for equilibrium systems to the case of non-equilibrium alloys. The method developed is used to simulate $\mathrm{A} 1 \rightarrow \mathrm{L}_{2}$ and $\mathrm{A} 1 \rightarrow \mathrm{A} 1+\mathrm{L}_{2}$ transformations after a quench of an alloy from the disordered $\mathrm{A} 1$ phase to the single-phase $\mathrm{L}_{2}$ state or the two-phase $\mathrm{A} 1+\mathrm{L}_{2}$ state for a number of alloy models with both short-range and long-range interactions. Simulations of the $\mathrm{A} 1 \rightarrow \mathrm{L}_{2}$ transition show a sharp dependence of the microstructural evolution on the type of interaction, and particularly on the interaction range. The simulations also reveal a number of peculiar features in both the transient microstructures and the transformation kinetics, many of them agreeing well with experimental observations. Microstructural evolution under $\mathrm{A} 1 \rightarrow \mathrm{A} 1+\mathrm{L}_{2}$ transition was found to be less sensitive to the type of the finite-range ('chemical') interaction, while in the presence of elastic interaction this evolution shows a number of specific features which were earlier discussed phenomenologically by Khachaturyan and co-workers and are illustrated by our simulations. We also consider the problem of the occurrence of a transient congruent ordering under $\mathrm{A} 1 \rightarrow \mathrm{A} 1+\mathrm{L}_{2}$ transformation and discuss the microstructural features of this stage.
\end{abstract}

\section{Introduction}

Problems of evolution of non-equilibrium statistical systems attract attention in many areas of physics. These problems are of particular interest for configurational alloy kineticsthe evolution of the atomic distribution in non-equilibrium alloys. The microstructure and macroscopic properties of such alloys, e.g. strength and plasticity, depend crucially on their thermal and mechanical history-for example, on the kinetic path taken during phase transformations. Theoretical treatments of these problems usually employ either Monte Carlo simulation — see e.g. [1-3]—or various phenomenological kinetic equations for local concentrations [4-6]. However, Monte Carlo studies in this field are time consuming, and until now they provided limited information on the details of the microstructural evolution [1-3]. Use of the phenomenological kinetic equations is more feasible, and Khachaturyan and co-workers [4-6] used this approach as a basis for discussing many interesting microstructural effects. However, the phenomenological approach employs a number of unclear approximations - in particular, the extrapolation of linear Onsager equations for weakly non-equilibrium states to the non-linear region of states far from equilibrium, and the relation between the phenomenological and microscopic approaches is also often unclear $[7,8]$.

A consistent microscopical description of non-equilibrium alloys can be based on the fundamental master equation for the probabilities of various atomic distributions over lattice 
sites [7-18]. It was shown in $[13,16]$ that under the usual assumptions regarding the thermally activated atomic exchange mechanism [9-11], one can derive from the master equation an exact set of kinetic equations for local concentrations and correlators of their fluctuations. To solve these equations, one can employ various approximate methods analogous to those used in equilibrium statistical physics. The simplest method is that of the kinetic mean-field approximation (KMFA) which corresponds to a generalization of the mean-field approximation (MFA) of equilibrium statistical physics to the case of inhomogeneous and non-stationary distributions. The KMFA was used in most of the previous applications of the master equation approach to alloy kinetics $[7-12,18]$. However, to solve the exact kinetic equations, one can also use more accurate approximations, and references $[14,15,17]$ provide examples of treatments of various kinetic phenomena going beyond the KMFA.

The description of ordering phase transitions in FCC alloys such as the $\mathrm{L}_{2}$ and $\mathrm{L}_{0}$ orderings is a well known problem of equilibrium statistical physics where the simple MFA is insufficient, and more exact, cluster methods are necessary. The MFA yields qualitatively wrong phase diagrams for such systems, while the cluster-variation method (CVM) $[20,21,30]$ or its simplified version, the cluster-field method (CFM) [22, 23], can describe these phase diagrams rather accurately. Therefore, to microscopically describe the kinetics of such transitions, the kinetic generalizations of the CVM or the CFM are evidently needed. As was discussed in $[13,23]$, for the versions of the CVM usually employed, such as the tetrahedron or the tetrahedron-octahedron approximations [20,21], such generalizations seem to be cumbersome and are hardly suitable for application to non-uniform alloys. However, such kinetic generalization is possible and feasible for the simple version of the CFM discussed in [23], the tetrahedron cluster-field method (TCFM). In the present work we describe this generalization, the kinetic tetrahedron cluster-field method (KTCFM), and apply it to the studies of some problems of kinetics of $\mathrm{L}_{2}$-type orderings.

Features of microstructural evolution after a quench of an alloy from the disordered FCC phase (A1 phase) to the single-phase $\mathrm{L}_{2}$ or the two-phase $\mathrm{A} 1+\mathrm{L}_{2}$ area of the phase diagram have been studied by many authors; see e.g. [24-29]. In this work we discuss the two problems in that field which seem to attract particular interest. The first one concerns the relation between the effective interatomic interactions $v_{n}$ in an alloy and the characteristic morphologies and evolution of antiphase or interphase boundaries (APBs or IPBs). Numerous observations show that in some $\mathrm{L}_{2}$-ordered alloys $\mathrm{APBs}$ are predominantly oriented along certain crystallographic directions, usually in (100) planes, while in other alloys this tendency is less pronounced or absent [24-26]. The driving force responsible for the APB orientation has not yet been explained [24]. It was shown by Kikuchi and Cahn [30] that for the nearestneighbour interaction model certain APBs, called conservative ones and lying in the (100) planes, have zero surface energy, while for all other APBs this energy is positive. Therefore, a strong (100) alignment of APBs observed in some alloys was qualitatively explained as the manifestation of a predominantly nearest-neighbour interaction in these systems [24, 25]. However, in most real alloys the non-nearest-neighbour interactions are quite significant $[21,31,32]$, and their effect on the morphology and evolution of APBs and IPBs has not yet been studied. In this work we investigate these problems using the KTCFM to simulate $\mathrm{A} 1 \rightarrow \mathrm{L}_{2}$ and $\mathrm{A} 1 \rightarrow \mathrm{A} 1+\mathrm{L}_{2}$ transformations for a number of alloy models with both short-range and long-range interactions. Our simulations show, in particular, that the type of the microstructural evolution under the $\mathrm{A} 1 \rightarrow \mathrm{L}_{2}$ transition sharply depends on the form of the effective interactions $v_{n}$. In the systems with a relatively short-range interaction, such as the second-neighbour interaction models, the energies of the conservative APBs remain small and the tendency of formation of such APBs under the $\mathrm{A} 1 \rightarrow \mathrm{L}_{2}$ transition is quite pronounced. In contrast, in the systems with an extended interaction range-for example, in 
the fourth-neighbour interaction models treated below-the energies of the conservative and non-conservative APBs are close to each other and the conservative APBs play a minor or no role in the transient microstructures. Therefore, a comparison of simulated microstructures with the experimentally observed ones can provide information about the interactions $v_{n}$ in real alloys, which can complement the estimates of $v_{n}$ obtained by other methods $[21,31,32]$.

We also discuss the problem of 'transient congruent ordering' under alloy decomposition with ordering which has recently received some attention [1,2,27-29]. This problem was first discussed by Allen and Cahn [33], who supposed that such a transient state should be the first stage of evolution after a quench of a disordered alloy below the ordering spinodal (the instability limit of the disordered phase with respect to ordering) into the two-phase field of coexistence of ordered and disordered phases. Allen and Cahn argued that such ordering at an unchanged initial composition (congruent ordering) requires only local atom exchanges, whereas evolution of the composition needs long-range atom transport with much longer times. This congruent ordering should result in a microstructure of antiphase microdomains. Then this transient state is succeeded by a decomposition of an alloy which is mainly realized by the 'wetting' of newly formed APBs with the disordered phase, leading to two-phase morphology with layers of disordered phase separating ordered antiphase domains [33]. These considerations were supported by a number of experimental observations, mainly for BCC-based alloys [29,33], as well as by the computer simulations of Khachaturyan and coworkers $[4,5]$ based on the above-mentioned phenomenological kinetic equations. However, in a careful study of the $\mathrm{A} 1 \rightarrow \mathrm{A} 1+\mathrm{L}_{2}$ transformation in $\mathrm{Al}-\mathrm{Li}$ alloys, Haasen and coworkers [27] observed some microstructures which these authors considered as incompatible with congruent ordering. Recent Monte Carlo simulations [1,2] as well as the small-angle scattering data [28] did not provide evidence for congruent ordering under $\mathrm{A} 1 \rightarrow \mathrm{A} 1+\mathrm{L} 1_{2}$ transitions either. Therefore, this problem is considered somewhat controversial [29]. In this work we use the KTCFM to simulate $\mathrm{A} 1 \rightarrow \mathrm{A} 1+\mathrm{L}_{2}$ transformation under conditions similar to those of the experiments in [27]. Our simulation confirms the presence of congruent ordering, while the microstructures observed by Haasen and co-workers [27] can be explained by some secondary effects illustrated by our simulation.

The paper is organized as follows. In section 2 we describe the kinetic tetrahedron clusterfield method. In section 3 we discuss the models and methods of simulation employed. In section 4 we describe our simulations of the $\mathrm{A} 1 \rightarrow \mathrm{L1}_{2}$ transformation for a number of interaction models. In section 5 we describe similar simulations of the $\mathrm{A} 1 \rightarrow \mathrm{A} 1+\mathrm{L} 1_{2}$ transition and also discuss the influence of elastic forces on microstructural evolution. Then we consider the congruent ordering problem simulating $\mathrm{A} 1 \rightarrow \mathrm{A} 1+\mathrm{L}_{2}$ transition at concentrations not far from the ordering spinodal, which corresponds to the conditions of the experiments of Haasen and co-workers [27]. Our main conclusions are summarized in section 6.

\section{The kinetic tetrahedron cluster-field method}

We consider a binary substitutional alloy A-B. Various distributions of atoms over lattice sites $i$ are described by the sets of occupation numbers $\left\{n_{i}\right\}$, where the operator $n_{i}=n_{\mathrm{A} i}$ is unity when the site $i$ is occupied by atom A and zero otherwise. The interaction Hamiltonian $H$ has the form

$$
H=\sum_{i>j} v_{i j} n_{i} n_{j}+\sum_{i>j>k} v_{i j k} n_{i} n_{j} n_{k}+\cdots
$$


where $v_{i \ldots j}$ are effective interactions, and for simplicity below we consider the pair interaction model for which equation (1) includes only the first term.

The general form of the master equation approach was described in references [13] and [16], to be referred to as I and II. Below, we present the main equations of this approach that are used in what follows. The fundamental master equation for the probability $P$ of finding the occupation number set $\left\{n_{i}\right\}=\alpha$ is

$$
\mathrm{d} P(\alpha) / \mathrm{d} t=\sum_{\beta}[W(\alpha, \beta) P(\beta)-W(\beta, \alpha) P(\alpha)] \equiv \hat{S} P
$$

where $W(\alpha, \beta)$ is the $\beta \rightarrow \alpha$ transition probability.

It has been shown in [16] that in the studies of the advanced stages of phase transformations (which are being considered in the present work), the true vacancy-mediated atomic exchange mechanism can be replaced by an equivalent direct-exchange model, which results in great simplification of the calculations. Adopting also the thermally activated atomic exchange model [9-11] for probabilities $W(\alpha, \beta)$, we can express the transfer matrix $\hat{S}$ in equation (2) in terms of the probability $W_{i j}^{\mathrm{AB}}$ of an elementary intersite exchange ('jump') $\mathrm{A} i \leftrightarrow \mathrm{B} j$ (see [11] for details):

$$
W_{i j}^{\mathrm{AB}}=n_{i} n_{j}^{\prime} \omega_{i j} \exp \left[-\beta\left(E_{i j}^{s}-\hat{E}_{i j}^{i n}\right)\right] \equiv n_{i} n_{j}^{\prime} \gamma_{i j} \exp \left(\beta \hat{E}_{i j}^{i n}\right) .
$$

Here: $n_{j}^{\prime}=n_{\mathrm{B} j}=1-n_{j} ; \omega_{i j}$ is the attempt frequency; $\beta=1 / T$ is the reciprocal temperature; $E_{i j}^{s}$ is the saddle-point energy; $\gamma_{i j}=\omega_{i j} \exp \left(-\beta E_{i j}^{s}\right)$ is the configurationally independent factor in the jump probability; and $\hat{E}_{i j}^{i n}$ is the initial (before the jump) configurational energy of jumping atoms given by equations (I.4) or (II.7).

Multiplying equation (2) by operators $n_{i}, n_{i} n_{j}$, etc, and summing over all configurational states, i.e. over all number sets $\left\{n_{i}\right\}$, we obtain the set of equations for averages $\left\langle n_{i} n_{j} \cdots n_{k}\right\rangle \equiv$ $g_{i j \cdots k}$, and in particular, for the mean occupation $c_{i}=\left\langle n_{i}\right\rangle=g_{i}$ :

$$
\mathrm{d} g_{i j \cdots k} / \mathrm{d} t=\left\langle n_{i} n_{j} \cdots n_{k} \hat{S}\right\rangle
$$

where $\langle(\cdots)\rangle=\operatorname{Tr}\{(\cdots) P\}$ means averaging over the distribution $P$, i.e. the summation of the operator product $(\cdots) P$ over all occupation number sets $\{\alpha\}$.

Since the $n_{i}$ are the projection operators $\left(n_{i}^{2}=n_{i}\right)$, the most general expression for the distribution function $P(\alpha)=P\left\{n_{i}\right\}$ in (2) can be written as

$$
P\left\{n_{i}\right\}=\exp \left[\beta\left(\Omega+\sum_{i} \lambda_{i} n_{i}-Q\right)\right] \equiv \exp \left[\beta\left(\Omega-Q^{\prime}\right)\right] .
$$

Here the quantities $\lambda_{i}$ can be called the 'local chemical potentials', the 'quasi-Hamiltonian' $Q^{\prime}$ is

$Q^{\prime}=-\sum_{\mathrm{i}} \lambda_{i} n_{i}+Q=-\sum_{i} \lambda_{i} n_{i}+\sum_{i>j} a_{i j} n_{i} n_{j}+\sum_{i>j>k} a_{i j k} n_{i} n_{j} n_{k}+\cdots$

and the 'quasi-interaction' term $Q$ in $(6 a)$ is an analogue of the interaction Hamiltonian $H$ in (1). Also, the generalized grand canonical potential $\Omega$ is determined by the normalizing condition

$$
\Omega=-T \ln \operatorname{Tr} \exp \left(-\beta Q^{\prime}\right) .
$$

Note that according to equation (6b) the mean occupation $c_{i}$ is related to $\Omega\left\{\lambda_{i}\right\}$ as

$$
c_{i}=\partial \Omega / \partial \lambda_{i} .
$$

The basic approximation of the kinetic cluster-field approach (KCFA) is neglecting the interaction renormalization effects in the distribution function $P$ in equation (5), i.e. putting 
the quasi-interactions $a_{i \ldots j}$ (which are, generally, both time and space dependent) equal to the interaction constants $v_{i \ldots j}$ in the Hamiltonian (1). For some specific problems-in particular, for the finding of the so-called off-diagonal Onsager terms in the diffusivities- the KCFA turns out to be insufficient, and more refined methods should be used [17]. However, one may expect this approximation to adequately describe the main kinetic features of the phase transformation if the version of the CFM employed describes its thermodynamics well. It was shown in reference [23] that a relatively simple version of the CFM, the TCFM discussed in that work, provides a rather accurate description of the phase diagrams with $\mathrm{L} 1_{2}$ and $\mathrm{L} 1_{0}$ orderings for a number of realistic alloy models. Therefore, one may expect a kinetic generalization of this approach to adequately describe the kinetics of such orderings.

The generalized free energy $F$ defined by general equations (I.24) or (II.23) takes a simplified form in the KCFA:

$$
F=\Omega+\sum_{i} \lambda_{i} c_{i}
$$

Using equation (7), we find that the local chemical potential $\lambda_{i}$ is related to $F=F\left\{c_{i}\right\}$ as

$$
\lambda_{i}=\partial F / \partial c_{i} .
$$

General kinetic equations (15) in the KCFA (as well as in the KMFA) are reduced to the set of equations (I.25) or (II.30) for mean occupations $c_{i}$ :

$$
\mathrm{d} c_{i} / \mathrm{d} t=2 \sum_{j} M_{i j} \sinh \left[\beta\left(\lambda_{j}-\lambda_{i}\right) / 2\right] .
$$

Here $M_{i j}$ is the generalized mobility, which for the pair interaction model employed is given by equation (II.32b):

$M_{i j}=\gamma_{i j}\left\langle n_{i}^{\prime} n_{j}^{\prime} \exp \left\{\frac{1}{2} \beta\left[\lambda_{i}+\lambda_{j}-\sum_{k}\left(v_{i k}+v_{j k}\right) n_{k}+\sum_{k}\left(u_{i k}+u_{j k}\right) n_{k}\right]\right\}\right\rangle$

where $\gamma_{i j}, n_{i}^{\prime}$ and $v_{i j}=V_{i j}^{\mathrm{AA}}-2 V_{i j}^{\mathrm{AB}}+V_{i j}^{\mathrm{BB}}$ are the same as in equations (3) and (1), while $u_{i j}=V_{i j}^{\mathrm{AA}}-V_{i j}^{\mathrm{BB}}$ is the so-called asymmetrical potential [9]. Note that the mobility $M_{i j}$ in equation (10) is just a kinetic factor which affects only the timescale, while the difference $\lambda_{j}-\lambda_{i}$ is the generalized driving force which determines all trends in the microstructural evolution.

In actual calculations, both quantities $\lambda_{i}$ and $M_{i j}$ in (10) should be found using some particular approximation of the CFM or the MFA. As was mentioned in section 1, a correct description of orderings in FCC alloys requires that the free energy $F\left\{c_{i}\right\}$ and its derivatives $\lambda_{i}=\partial F / \partial c_{i}$ are found using the cluster methods, so as to take into account the strong nearestneighbour correlations in such alloys $[20-23,30]$. However, in the mobility $M_{i j}$, equation (11), these correlations seem to result only in some quantitative factors that weakly depend on the local composition and ordering and do not lead to qualitative effects. Therefore, in the studies of the main features of microstructural evolution, it does not seem necessary to take into account the above-mentioned correlation effects in $M_{i j}$, and the simple MFA can be sufficient. Therefore, in this work we employ the KMFA expression (II.36) for the mobility $M_{i j}$ :

$$
M_{i j}=\gamma_{i j}\left[c_{i} c_{j} c_{i}^{\prime} c_{j}^{\prime} \exp \left(\beta u_{i}+\beta u_{j}\right)\right]^{1 / 2}
$$

where $c_{i}^{\prime}=c_{\mathrm{B} i}=1-c_{i}$.

To find the generalized driving force $\lambda_{j}-\lambda_{i}$ in (10), one should find the local chemical potential $\lambda_{i}$. To calculate $\lambda_{i}\left\{c_{j}\right\}$ for a non-uniform alloy we employ the tetrahedron clusterfield method discussed in reference [23], to be referred to as III. In this method the thermodynamic contribution of the nearest-neighbour interaction $v_{1}$ is described using the division 
of the FCC lattice into 'non-overlapping' tetrahedron clusters with common vertices and no common edges, which was suggested by Yang [34] in his 'quasi-chemical' method, while the contributions of non-nearest-neighbour interactions $v_{n}$ with $n>1$ are taken into account in the pair cluster approximation and are written out analytically [22,23]. Thus the local chemical potential $\lambda_{i}$ is given $\dagger$ by equation (III.41):

$$
\lambda_{i}=\ln \frac{c_{i}}{1-c_{i}}+\sum_{j, n>1} \tilde{\mu}_{i}^{i j, n}+\sum_{\{j k l\} \in t Y, i} \tilde{\mu}_{i}^{i j k l}
$$

where the second and the third terms describe the contributions of non-nearest-neighbour and nearest-neighbour interactions, respectively.

The quantity $\tilde{\mu}_{i}^{i j, n}$ in the second term corresponds to the interaction $v_{i j}=v_{n}$ between sites $i$ and $j$ which are $n$th neighbours in the lattice:

$$
\tilde{\mu}_{i}^{i j, n}=T \ln \left(1-c_{j} g_{i j}\right)
$$

where $g_{i j}$ is expressed in terms of the Mayer function $f_{i j}=\exp \left(-\beta v_{i j}\right)-1$ as follows:

$g_{i j}=\frac{2 f_{i j}}{R_{i j}+1+f_{i j}\left(c_{i}+c_{j}\right)} \quad R_{i j}=\left\{\left[\left(1+\left(c_{i}+c_{j}\right) f_{i j}\right]^{2}-4 c_{i} c_{j} f_{i j}\left(f_{i j}+1\right)\right\}^{1 / 2}\right.$.

In the case of weak interaction, $\beta v_{i j} \ll 1$, equation (14) transforms into the result from the mean-field approximation: $\tilde{\mu}_{i}^{i j, n}=v_{i j} c_{j}$.

The quantity $\tilde{\mu}_{i}^{i j k l}$ in the last sum of equation (13) corresponds to the contribution to $\lambda_{i}$ of Yang's tetrahedron of sites $i, j, k, l$, and the notation $\{j k l\} \in t Y, i$ means that the summation is performed over four Yang tetrahedra that contain site $i$. The expression for $\tilde{\mu}_{i}^{i j k l}$ is given by equation (III.42):

$$
\tilde{\mu}_{i}^{i j k l}=\ln \left[y_{i}^{i j k l}\left(1-c_{i}\right) / c_{i}\right] .
$$

Here the quantity $y_{i}^{i j k l}$ is defined by the relations generalizing equations (III.27)-(III.29) to the case of the distribution (5) with the KCFA approximation $Q=H$. Denoting for brevity the tetrahedron cluster of sites $i, j, k, l$ as $\alpha$, we can write the KCFA generalization of equation (III.27) as

$$
y_{i}^{\alpha}=\exp \left[\beta\left(\lambda_{i}-\psi_{i}^{\alpha}\right)\right]
$$

where $\psi_{i}^{\alpha}$ is the effective field of the environment acting on site $i$ in the cluster $\alpha$ [23].

Equations determining the quantities $y_{i}^{\alpha}$ in terms of intra-cluster mean occupations $c_{s}$ with $s$ equal to $i, j, k$ or $l$ can be written out with the use of the cluster partition function $Z_{\alpha}$. The latter corresponds to an obvious generalization of equation (III.4):

$$
Z_{\alpha}=\operatorname{Tr} \exp \left(-\beta Q_{\alpha}^{\prime}\right)
$$

where $Q_{\alpha}^{\prime}$ is the cluster quasi-Hamiltonian analogous to the cluster Hamiltonian (III.5):

$$
Q_{\alpha}^{\prime}=\sum_{s}\left(\psi_{s}^{\alpha}-\lambda_{s}\right) n_{s}+\sum_{s<s^{\prime}} v_{s s^{\prime}} n_{s} n_{s^{\prime}}
$$

and the $v_{s s^{\prime}}=v_{1}$ correspond to intra-cluster interactions. Using equations (17)-(19) and (7), we can express the mean occupation $c_{s}$ in terms of $y_{s^{\prime}}^{\alpha}$ just as in equation (III.29):

$$
c_{s}=y_{s}^{\alpha} \partial \ln Z_{\alpha} / \partial y_{s}^{\alpha} .
$$

$\dagger$ Note the misprints in equations (40) and (41) of [23]: the subscripts ' $n>2$ ' should be changed to: ' $n>1$ '. Also note that the inappropriate term 'quasi-chemical tetrahedron cluster method (QCTCM)' in the English translation of the paper [23] should be read as: 'tetrahedron cluster-field method (TCFM)' throughout that paper. 
According to equation (18), the cluster partition function $Z_{\alpha}$ is a polynomial in $y_{s}^{\alpha}$ :

$Z_{\alpha}=1+\sum_{s} y_{s}^{\alpha}+\zeta \sum_{s<s^{\prime}} y_{s}^{\alpha} y_{s^{\prime}}^{\alpha}+\zeta^{3} \sum_{s<s^{\prime}<s^{\prime \prime}} y_{s}^{\alpha} y_{s^{\prime}}^{\alpha} y_{s^{\prime \prime}}^{\alpha}+\zeta^{6} y_{i}^{\alpha} y_{j}^{\alpha} y_{k}^{\alpha} y_{l}^{\alpha}$

where $\zeta=\exp \left(-\beta v_{1}\right)$. Therefore, the right-hand side of equation (20) is the ratio of two polynomials in $y^{\alpha}$, where the numerator includes only those terms of the denominator which contain the factor $y_{s}^{\alpha}$. For all clusters $\alpha$, the equation system (20) can easily be solved for the functions $y_{i}^{\alpha}$ using Newton's method.

Equations (10), (12) and (13), together with (14) and (15) for $\tilde{\mu}_{i}^{i j, n}$ and (16) and (20) for $\tilde{\mu}_{i}^{i j k l}$, provide a closed set of equations for finding the mean occupations $c_{i}(t)$. This method for the calculation of $c_{i}(t)$ will hereafter be called 'the kinetic tetrahedron cluster-field method'.

\section{Models and methods of simulation}

Below, we use the above-described KTCFM to simulate $\mathrm{A} 1 \rightarrow \mathrm{L}_{2}$ and $\mathrm{A} 1 \rightarrow \mathrm{A} 1+\mathrm{L}_{2}$ transformations. For this simulation we employ five alloy models with the following values of the effective pair interactions $v_{n}$ :

(1) The second-neighbour interaction model with $v_{2} / v_{1}=\epsilon=-0.125$.

(2) The same model with $\epsilon=-0.25$.

(3) The same model with $\epsilon=-0.5$.

(4) The fourth-neighbour interaction model with $v_{n}$ estimated by Chassagne et al [35] from their experimental data for the $\mathrm{Ni}_{0.927} \mathrm{Al}_{0.073}$ alloy at $T=673 \mathrm{~K}: v_{1}=1680 \mathrm{~K}$, $v_{2}=-210 \mathrm{~K}, v_{3}=35 \mathrm{~K}$ and $v_{4}=-207 \mathrm{~K}$.

(5) The fourth-neighbour interaction model with $v_{2} / v_{1}=-0.5, v_{3} / v_{1}=0.25$ and $v_{4} / v_{1}=$ -0.125 .

The effective interaction range for these models increases monotonically with the model number. Therefore, a comparison of the simulation results for these models will provide information on the effect of the interaction range on the microstructural evolution. Phase diagrams for models $1,2,3$ and 4 have been calculated in reference [23]. It was found that the phase diagrams calculated with the help of the above-mentioned simple TCFM are very close to those obtained using rather accurate and sophisticated versions of the CVM [20,21], namely the tetrahedron-octahedron CVM for the ordered phase and the double-tetrahedronoctahedron CVM for the disordered phase. Figure 1 illustrates this similarity for models 2 and 4; for models 1 and 3 both phase diagrams and their agreement with the CVM are similar.

In our treatment of $\mathrm{A} 1 \rightarrow \mathrm{A} 1+\mathrm{L}_{2}$ transformation we also discuss the influence of elastic forces on microstructural evolution. To this end we consider two more models, to be denoted as $2^{\prime}$ and $4^{\prime}$, which correspond to the addition of the elastic interaction $v^{e l}$ to the above-mentioned 'short-range', or 'chemical' interactions $v_{n}=v_{n}^{c}$ of the corresponding model 2 or 4 , and for model $2^{\prime}$ we put: $v_{1}^{c}=1000 \mathrm{~K}, v_{2}^{c}=-v_{1} / 4=-250 \mathrm{~K}$. For the elastic interaction $v^{e l}$ we use the model described in [18] for the two-dimensional (2D) square lattice, but reformulated for the three-dimensional (3D) FCC lattice. This form of $v^{\text {el }}$ corresponds to the general expression suggested by Khachaturyan [36,37], but the phonon dynamical matrix in that expression is described by a Born-von Karman model with the first- and secondneighbour force constants only, and the second-neighbour constants are supposed to correspond to a spherically symmetrical interaction [18]. This model includes three independent force constants which are expressed in terms of elastic constants $c_{i k}$. The $c_{i k}$-values, the lattice constant $a$ and the concentration expansion coefficient $u_{0}=\mathrm{d} \ln a / \mathrm{d} c$ entering the expression 

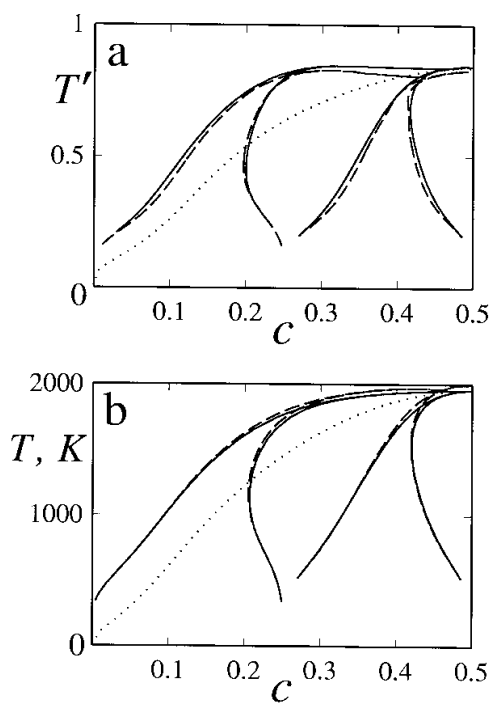

Figure 1. (a) The phase diagram in the $c, T^{\prime}$-plane for the second-neighbour-interaction model 2 with $\epsilon=v_{2} / v_{1}=-0.25$ where $T^{\prime}=T / v_{1}$ is the reduced temperature. The solid and dashed lines are curves of equilibrium between phases, $T_{e}(c)$, calculated in [23] using the TCFM and CVM, respectively, as described in the text. The regions separated by the lines $T_{e}(c)$ correspond to the following phases (from left to right): disordered $\mathrm{A}_{1}$ phase; coexistence of $\mathrm{A}_{1}$ and $\mathrm{L} 1_{2}$ phases; $\mathrm{L}_{2}$ phase; coexistence of $\mathrm{L}_{2}$ and $\mathrm{L} 1_{0}$ phases; $\mathrm{L}_{0}$ phase. The dotted line is the ordering spinodal $T_{s}(c)$ calculated using the TCFM. (b) The phase diagram in the $c, T$-plane for the model 4 with $v_{n}$ estimated from experiments for $\mathrm{Ni}-\mathrm{Al}$ alloys [35]. The lines have the same meaning as in (a).

for $v^{e l}$ in our simulations correspond to the FCC Ni-Al alloys [35, 38]: $c_{11}=2.23 \mathrm{Mbar}$, $c_{12}=1.47 \mathrm{Mbar}, c_{44}=1.25 \mathrm{Mbar}, a=3.57 \AA$ and $u_{0} \simeq 0.05$.

Let us discuss the distributions of mean occupations $c_{i}$ under $\mathrm{L} 1_{2}$ or $\mathrm{L} 1_{0}$ ordering. For a homogeneous ordered structure this distribution has the form [36]

$$
c_{i}=c+\eta_{1} \exp \left(\mathrm{i} \boldsymbol{k}_{1} \cdot \boldsymbol{R}_{i}\right)+\eta_{2} \exp \left(\mathrm{i} \boldsymbol{k}_{2} \cdot \boldsymbol{R}_{i}\right)+\eta_{3} \exp \left(\mathrm{i} \boldsymbol{k}_{3} \cdot \boldsymbol{R}_{i}\right)
$$

where $\boldsymbol{R}_{i}$ is the FCC lattice vector for site $i ; \eta_{1}, \eta_{2}$ and $\eta_{3}$ are three components of the vector order parameter; and $\boldsymbol{k}_{\alpha}$ is the superstructure vector corresponding to $\eta_{\alpha}$ :

$$
k_{1}=(1,0,0) 2 \pi / a \quad k_{2}=(0,1,0) 2 \pi / a \quad k_{3}=(0,0,1) 2 \pi / a .
$$

For the $L 1_{2}$ structure $\left|\eta_{1}\right|=\left|\eta_{2}\right|=\left|\eta_{3}\right|$, while four possible types of ordered domain correspond to the relations $\eta_{1}=\eta_{2}=\eta_{3}>0, \eta_{1}=-\eta_{2}=-\eta_{3}>0,-\eta_{1}=\eta_{2}=-\eta_{3}>0$ and $-\eta_{1}=-\eta_{2}=\eta_{3}>0$. In the $\mathrm{L} 1_{0}$ structure only one component $\eta_{\alpha}$ is present, which is either positive or negative; thus six types of ordered domain are possible.

To describe the inhomogeneous states of a partially ordered alloy-in particular, the antiphase boundaries-it is convenient to define 'local order parameters' which correspond to a spatial averaging over some local region. As was shown in [18] for the case of $\mathrm{D}_{3}$ ordering, a suitable description is provided by 'site-centred' local order parameters for which the averaging is taken over some nearest neighbourhood of each site $i$ while its occupation $c_{i}$ makes the largest contribution to the averages. For the $\mathrm{L}_{2}$ and $\mathrm{L} 1_{0}$ orderings, such site-centred squared local order parameters $\eta_{\alpha i}^{2}$ and local concentrations $\bar{c}_{i}$ can be defined as follows:

$$
\eta_{\alpha i}^{2}=\frac{1}{16}\left[c_{i}+\frac{1}{4} \sum_{j=n n(i)} c_{j} \exp \left(\mathrm{i} \boldsymbol{k}_{\alpha} \cdot R_{j i}\right)\right]^{2}
$$




$$
\bar{c}_{i}=\frac{1}{4}\left(c_{i}+\frac{1}{4} \sum_{j=n n(i)} c_{j}\right)
$$

where $n n(i)$ means the summation over nearest neighbours of site $i, \boldsymbol{R}_{j i}$ is $\boldsymbol{R}_{j}-\boldsymbol{R}_{i}$ and the coefficients on the right-hand side are chosen such that in the homogeneous case (22) we would have: $\eta_{\alpha i}^{2}=\eta_{\alpha}^{2}, \bar{c}_{i}=c$.

From the experimental point of view, the description of alloy states in terms of mean local occupations $c_{i}$ corresponds to the high-resolution electron microscopy (HREM) images [24]. This ' $c_{i}$-representation' is convenient for describing the atomic-scale microstructures characteristic of the initial stages of phase transformations. The later stages correspond to the formation of extended ordered regions which are more conveniently described by the local order parameter distributions. These distributions are experimentally observed in the transmission electron microscopy (TEM) images where the reflection intensity is proportional to the squared value of the relevant order parameter [24]. Therefore, it is convenient to characterize the partial $\mathrm{L} 1_{2}$ ordering by the distribution of quantities $\eta_{i}^{2}=\eta_{1 i}^{2}+\eta_{2 i}^{2}+\eta_{3 i}^{2}$, and this distribution is called below the ' $\eta^{2}$-representation'.

Our simulations were performed in FCC simulation boxes of sizes $L^{2} \times H$ (where $L$ and $H$ are given in units of the lattice constant $a$ ), with periodic boundary conditions. We used both $3 \mathrm{D}$ simulations with $H=L$ and quasi-2D simulations with $H=1$. Employing the 2D models is a usual method for extending the maximum size of microstructures examined [4-6, 8, 18], but, below, the conclusions derived from 2D simulations are usually complemented with 3D simulations. The simulation methods were the same as in references $[7,8,16,18]$. In the simulations of both $\mathrm{A} 1 \rightarrow \mathrm{L} 1_{2}$ and $\mathrm{A} 1 \rightarrow \mathrm{A} 1+\mathrm{L}_{2}$ transformations, the initial as-quenched distribution $c_{i}(0)$ was characterized by its mean value $c$ and small random fluctuations $\delta c_{i}$; usually we used $\delta c_{i}= \pm 0.01$.

\section{Kinetics of the $\mathrm{A} 1 \rightarrow \mathrm{L1}_{2}$ transformation}

In this section we consider the microstructural evolution after a rapid quench of a disordered A1 phase into the single-phase $\mathrm{L}_{2}$ state. To this end we simulate this transformation for the above-described five alloy models at the stoichiometric initial concentration $c=0.25$. We also discuss the effect of non-stoichiometry on the evolution, considering it for model 2.

Some results of our simulation are presented in figures 2-9. Most of them correspond to 2D simulations in boxes of size $V_{b}=L^{2} \times H$ with $H=1$ and $L=64$ or $L=128$, but for model 1 we also carried out a 3D simulation with $L=H=50$. For the given $z$-coordinate $x_{3}$ (with $x_{3}=0$ for 2D simulation), each figure includes all FCC lattice sites lying in two adjacent planes, $z=x_{3} a$ and $z=\left(x_{3}+1 / 2\right) a$; thus the figure of area $L^{2}$ shows $4 L^{2}$ lattice sites. The values of the temperature $T$ at $c=0.25$ for the different models were chosen such that its ratio to the ordering spinodal temperature $T_{s}$ at $c=0.25$ was approximately the same for all models: $T / T_{s} \simeq 0.8$; thus the influences of the temperature on the evolution for the different models are similar. The distribution of initial fluctuations $\delta c_{i}$ for the given size of the simulation box was taken as the same for all models. Therefore, the microstructures shown in figures 2, 4, 5 and 7 for $V_{b}=64^{2} \times 1$ correspond to the same distribution of initial $\delta c_{i}$, just like the microstructures shown in figures 8 and 9 for $V_{b}=128^{2} \times 1$.

In the description of 3D microstructures in figure 3 , we present the distributions of 'tetrahedrally averaged' local order parameters $\eta_{j t}^{2}$ rather than the site-centred $\eta_{i}^{2}$ shown in other figures. The $\eta_{j t}^{2}$-distributions correspond to planes $x_{3}=n+1 / 4$ equidistant from the atomic planes $x_{3}=n$ and $x_{3}=n+1 / 2$, and the point $j$ corresponds to the centre of a tetrahedron of four nearest FCC lattice sites, while $\eta_{j t}^{2}$ is obtained by averaging of site-centred $\eta_{i}^{2}$-values for 


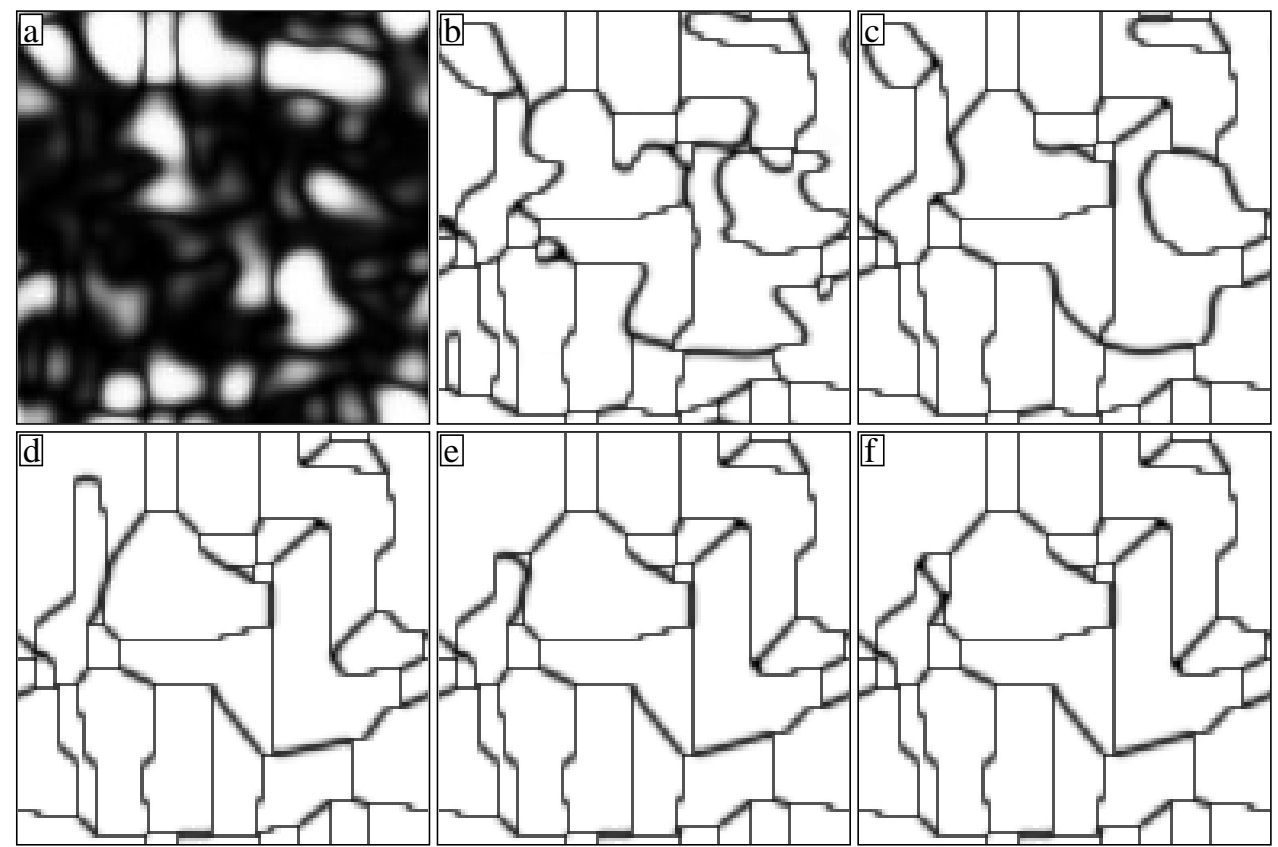

Figure 2. Temporal evolution of the second-neighbour-interaction model 1 with $\epsilon=v_{2} / v_{1}=$ -0.125 under the phase transformation $\mathrm{A} 1 \rightarrow \mathrm{L}_{2}$ shown in the $\eta^{2}$-representation for the simulation box size $V_{b}=64^{2} \times 1$ at $c=0.25, T^{\prime}=T / v_{1}=0.35$ and the following values of the reduced time $t^{\prime}=t \gamma_{n n}$ : (a) 2; (b) 3; (c) 20; (d) 100; (e) 177; and (f) 350. The grey level varies linearly with $\eta_{i}^{2}=\eta_{1 i}^{2}+\eta_{2 i}^{2}+\eta_{3 i}^{2}$ between its minimum and maximum values from completely dark to completely bright.

these four sites. We employ here this $\eta_{t}^{2}$-representation instead of the $\eta^{2}$-representation used in other figures since, as is discussed below, some APBs are locally $\mathrm{L} 1_{0}$ ordered, and when such an APB is situated in the plane of a figure (which is possible only for 3D simulations), its distribution of $\eta_{i}^{2}$ shows checkerboard-like irregularities. These irregularities are related just to our definition (24a) of local order parameters (which, for presentation of these particular microstructures, does not seem to be suitable), and to avoid such irregularities in figure 3 we employ the $\eta_{t}^{2}$-representation.

Let us first discuss the microstructures for model 1 with short-range interaction which are shown in figures 2 and 3. A distinctive feature of these microstructures is a predominance of conservative APBs with (100)-type orientation, particularly at the later stages of evolution shown in figures 2(f) and 3. As mentioned in section 1, for the nearest-neighbour interaction model with $v_{n>1}=0$, such APBs (for which the translation vector relating two ordered domains separated by this APB lies within the APB plane) have zero excess energy, unlike other, non-conservative APBs [30]. At finite small $\epsilon=v_{2} / v_{1}$, this energy is non-zero but small. Thus the above-mentioned predominance of conservative APBs for model 1 with small $|\epsilon| \simeq 0.1$ is natural. Figures 2 and 3 show that the conservative APBs are notably thinner than the non-conservative ones (at $\epsilon=0$ the width of a conservative APB is just one atomic layer [30]). The simulation also shows that the mobility of conservative APBs is quite low, and the microstructural evolution, i.e. the growth of ordered domains, is mainly realized via motion of non-conservative APBs; see frames 2(b)-2(f). Frame 2(a) shows an early stage of the transformation and illustrates that conservative APBs are already forming at this stage. 


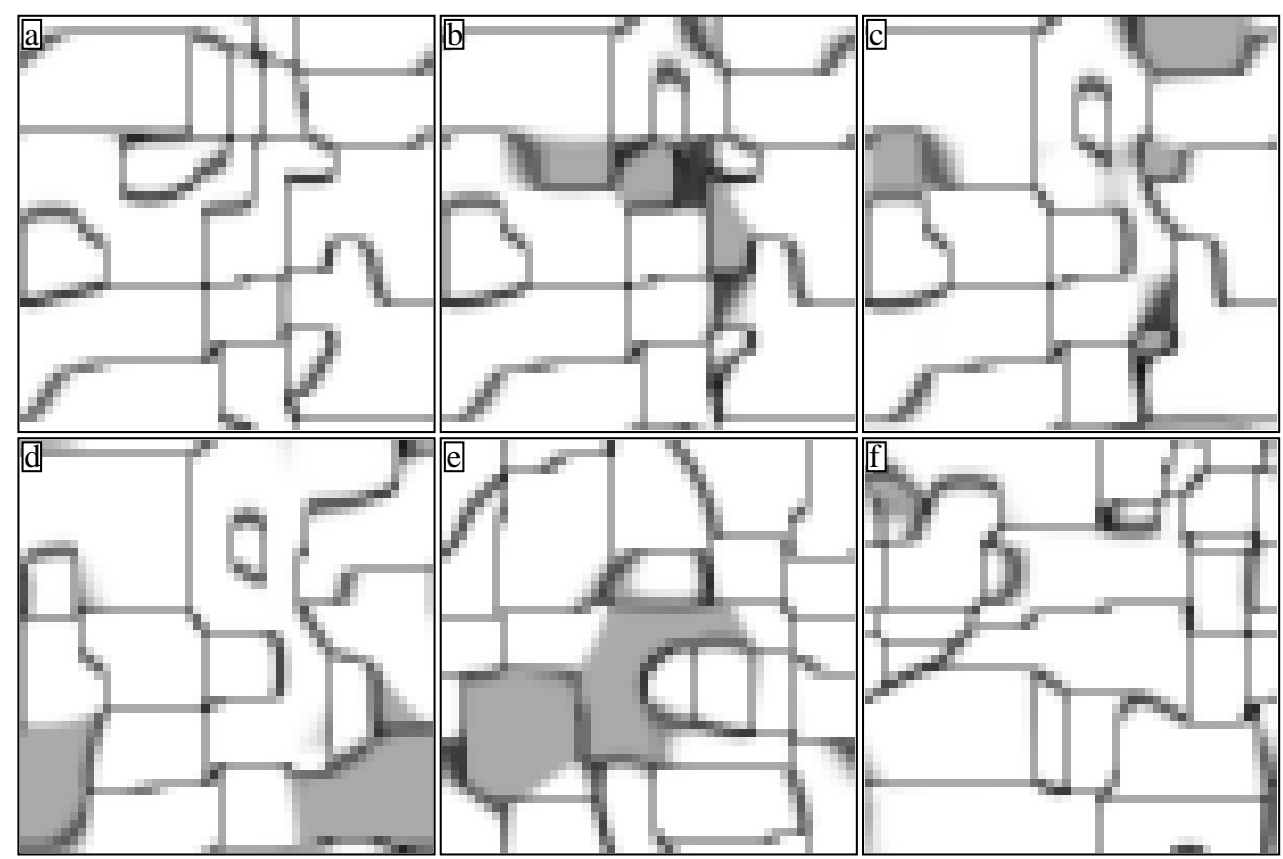

Figure 3. As figure 2, but shown in the $\eta_{t}^{2}$-representation described in the text for $V_{b}=50^{3}$ at $t^{\prime}=350$ and the following values of the coordinate $x_{3}=z / a$ : (a) $\frac{1}{4}$; (b) $2 \frac{1}{4}$; (c) $3 \frac{1}{4}$; (d) $6 \frac{1}{4}$; (e) $12 \frac{1}{4}$; and (f) $22 \frac{1}{4}$

Comparison of frames 2(f) and 3(a)-3(f) illustrates some characteristic differences between $2 \mathrm{D}$ and 3D microstructures. In particular, black or grey domains in frames 3(b)3 (f) correspond to locally disordered or locally $\mathrm{L}_{1}$-ordered APBs lying in the plane of figure, while in $2 \mathrm{D}$ microstructures only the edge-on APBs are presented, and for them local $\mathrm{L} 1_{0}$ orderings are not clearly seen in the $\eta^{2}$-representation used; see below. In addition, some 3D APBs in figure 3 are tilted with respect to the plane of the figure and thus seem to be thicker than the edge-on APBs shown in figure 2. However, the main features of the microstructure under 2D and 3D simulations appear to be the same, which justifies the employment of 2D simulations for the studies of these features.

The results shown in figures 2 and 3 can be compared with experimental observations for the $\mathrm{Cu}_{3} \mathrm{Au}$ system for which the nearest-neighbour interaction is supposed to exceed other interactions by an order of magnitude [25], just as for our model 1. In a detailed study of APBs in $\mathrm{Cu}_{3} \mathrm{Au}$, Loiseau and co-workers $[24,25]$ noted the following peculiar features in the APB distribution:

(i) The predominance of conservative APBs.

(ii) The presence of APBs with slight deviation from the (100) plane which actually have a step-like structure with small ledges normal to this plane.

(iii) The presence of triple junctions in which two conservative APBs normal to each other are connected with a non-conservative APB.

(iv) The presence of quadruple junctions in which a non-conservative APB connecting two triple junctions described in (iii) has an atomic-scale length.

(v) The presence of loop-like configurations of some non-conservative APBs adjacent to the conservative APBs. 


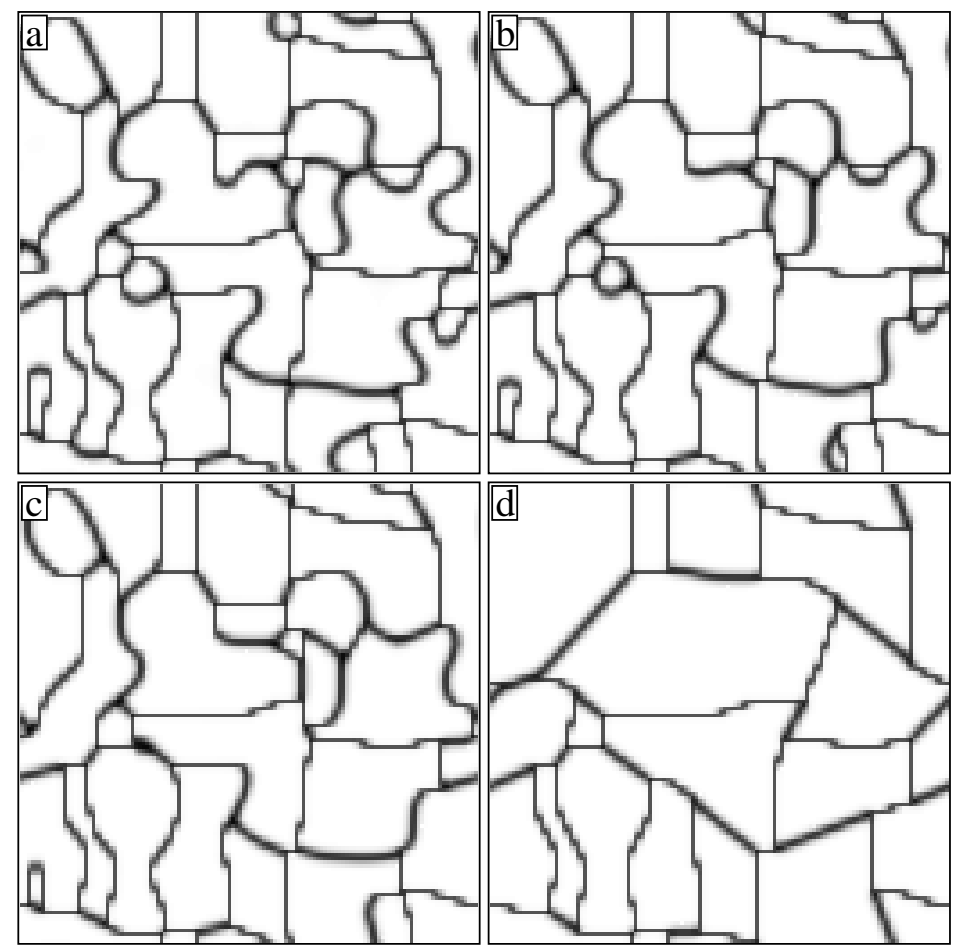

Figure 4. As figure 2, but for model 2 with $\epsilon=-0.25$ at $T^{\prime}=0.5$ and the following values of $t^{\prime}$ : (a) 3; (b) 7; (c) 10; and (d) 200 .

The microstructures shown in figures 2 and 3 can illustrate all of these observations, even though the experiments of Loiseau and co-workers $[24,25]$ correspond to much later stages of evolution and larger ordered domains. In particular: step-like APBs consisting of long conservative segments and the small non-conservative ledges mentioned in point (ii) are seen in frames 2(d), 3(d) and 3(f); triple and quadruple junctions mentioned in points (iii) and (iv) are present in almost all frames of figures 2 and 3; and loop-like non-conservative APBs adjacent to conservative APBs are seen in frames 3(e) and 3(f) (as well as in the upper right part of frames 4(a) and 4(b) and in the lower right part of frames 7(b) and 7(c)). Therefore, the above-mentioned features (i)-(v) of APBs in systems with short-range interaction appear to be already present at relatively early stages of the microstructural evolution.

Microstructures for models 2 and 3 are shown in figures 4-7. Model 2 with $|\epsilon|=\left|v_{2} / v_{1}\right|=$ 0.25 and model 3 with $|\epsilon|=0.5$ correspond to a larger effective interaction range compared to model 1 with $|\epsilon|=0.125$. Therefore, the above-discussed microstructural features related to the energetic preference of conservative APBs with respect to non-conservative ones are less pronounced for model 2 and still less so for model 3 . Thus the relative number of conservative APBs in the microstructures for these three models decreases with the increase of $|\epsilon|$, and these APBs in figures 4-7 are thicker than in figure 2. The reduction of the number of low-mobility conservative APBs leads to a notable acceleration of the microstructural evolution, which is clearly seen, in particular, from the comparison of frames 2(f), 4(d) and 7(d). However, for all these second-neighbour interaction models the conservative APBs still play an important role in the microstructures; thus all of these models can be considered as systems with a predominantly short-range interaction. 


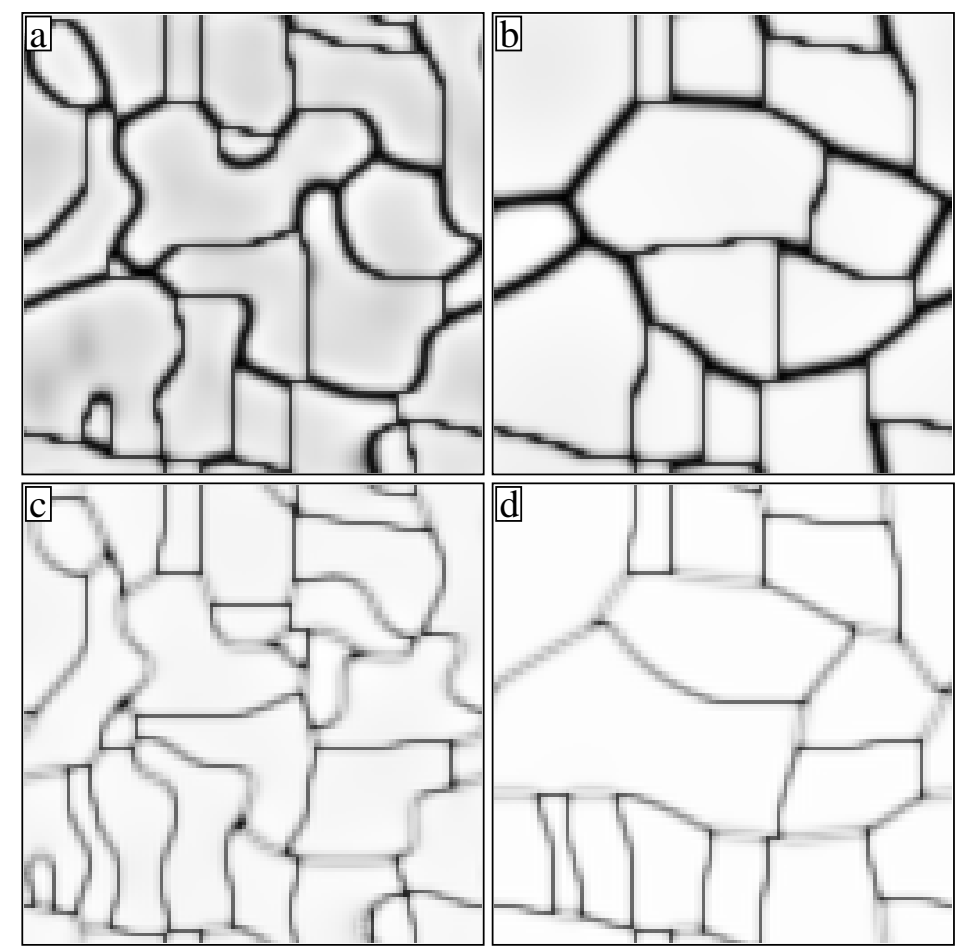

Figure 5. As figure 4, but at the following values of $c$ and $t^{\prime}$ : (a) $c=0.22, t^{\prime}=10$; (b) $c=0.22$, $t^{\prime}=200 ;$ (c) $c=0.32, t^{\prime}=10$; and (d) $c=0.32, t^{\prime}=200$.

Let us now consider the features of evolution of such systems. For the stoichiometric composition $c=0.25$, this evolution is illustrated by figures 2,4 and 7 . The figures show that the conservative APBs remain virtually immobile and the evolution is realized via motion of non-conservative APBs and their interaction with the conservative APBs. Let us discuss the mechanisms of this interaction, abbreviating for brevity the terms 'conservative APB', 'nonconservative APB' and 'antiphase ordered domain' to c-APB, nc-APB and APD, respectively. Examination of figures 2, 4 and 7 reveals the following characteristic processes of interaction between c-APBs and nc-APBs:

(a) 'Sweeping' of a $c-A P B$ by an adjacent moving $n c-A P B$. This process is seen: in the lefthand lower part of frames 4(a)-4(d) (as well as 7(a)-7(c) and 2(b), 2(c)); in the left-hand upper part of frames 2(d) and 2(e); in the right-hand upper part of frames 2(b) and 2(c); in frames 2(b)-2(d) at $x_{1}=x / a \simeq 50-55$ and $x_{2}=y / a \simeq 16-25$; etc.

(b) Wetting of a $c$-APB by adjacent $n c-A P B s$. This is seen: in frames 4(a)-4(c) (as well as 2(b), 2(c)) at $x_{1} \simeq 53, x_{2} \simeq 42$ where a horizontal c-APB is first wetted by adjacent left-hand and right-hand nc-APBs and is then 'consumed' by an nc-APB moving from below (with the disappearance of an APD lying between these two APBs); in the left-hand upper corner of frames 4(a)-4(c) (as well as 7(a)-7(c) and 2(b), 2(c)); etc.

(c) Motion of a triple junction of two nc-APBs with a $c-A P B$ along the direction of the latter up to the crossing of another $c-A P B$ normal to the first one, with the formation of a triple junction of these two $c-A P B s$ with an $n c-A P B$. Such a process is seen: in the right-hand upper part of frames 2(b) and 2(c) showing the formation of two vertical c-APBs ending with triple junctions; in frames 4(a)-4(d) (as well as 2(b), 2(c)) at $x_{1} \simeq 18, x_{2} \simeq 27$; in 


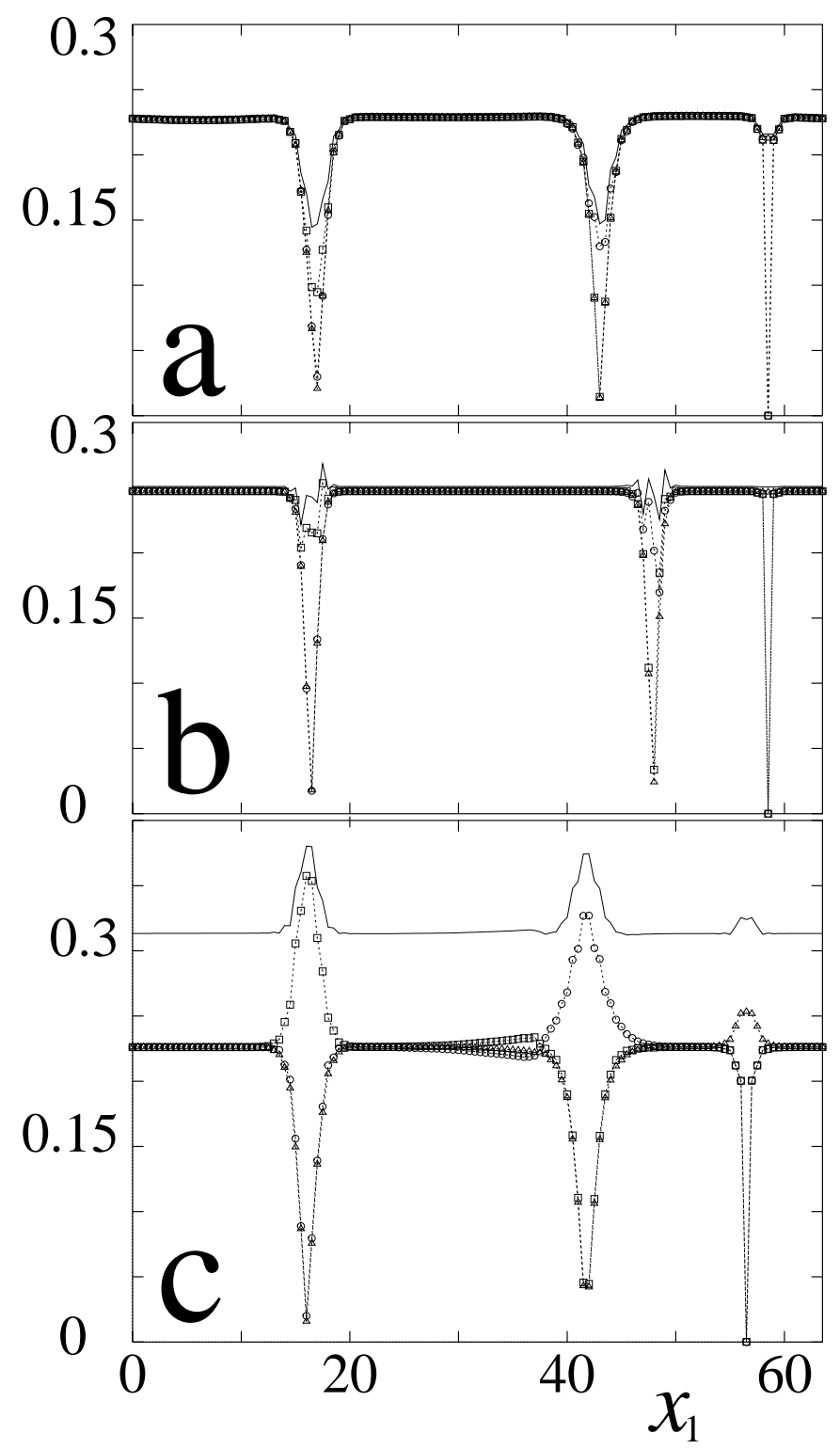

Figure 6. Variation of the local concentration $\bar{c}_{i}$ and local order parameters $\left|\eta_{\alpha i}\right|$ with the coordinate $x_{1}=x / a$ at $x_{2}=y / a=48$ for model 2 at $T^{\prime}=0.5, t^{\prime}=200$ and the following concentrations $c$ : (a) $c=0.22$ (frame 5(b)); (b) $c=0.25$ (frame 4(b)); and (c) $c=0.32$ (frame 5(d)). The solid line corresponds to $\bar{c}_{i}$; triangles, circles and squares correspond to $\left|\eta_{1 i}\right|,\left|\eta_{2 i}\right|$ and $\left|\eta_{3 i}\right|$, respectively; and dotted lines link identical symbols for clarity.

frames 4(a)-4(c) (as well as 2(b), 2(c)) at $x_{1} \simeq 61, x_{2} \simeq 22$; etc.

(d) 'Splitting' of an $n c-A P B$ into a $c-A P B$ and an $n c-A P B$ according to the reaction:

$$
n c-A P B \rightarrow c-A P B+n c-A P B
$$

with the formation of either a triple junction or a new APD. This peculiar kinetic process is observed for model 1 with the shortest interaction range, for which the c-APB energy 


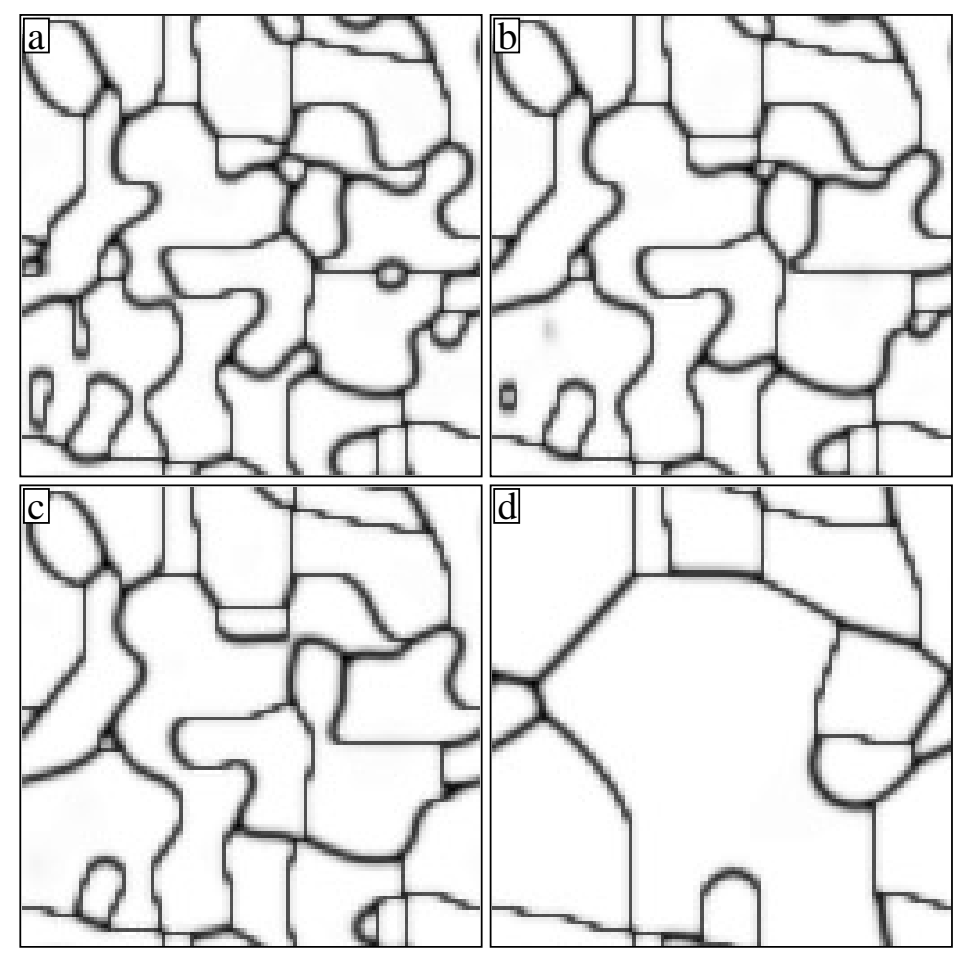

Figure 7. As figure 2, but for model 3 with $\epsilon=-0.5$ at $T^{\prime}=0.77$ and the following values of $t^{\prime}$ : (a) 3; (b) 5; (c) 7; and (d) 200 .

is particularly small. Splitting with the formation of triple junctions is seen: in the central lower part of frames 2 (c) and 2 (d) at $x_{1} \simeq 30, x_{2} \simeq 20$, and in the right-hand upper part of frames 2(b) and 2(c) at $x_{1} \simeq 48, x_{2} \simeq 45$. Splitting with the formation of a new domain is seen: in frames 2 (b) and 2(c) at $x_{1} \simeq 36, x_{2} \simeq 43$; in frames 2(c) and 2(d) at $x_{1} \simeq 12, x_{2} \simeq 35$; and in frames 2 (d)-2(f) at $x_{1} \simeq 12, x_{2} \simeq 42$. The latter process seems to be particularly interesting and includes several stages. First, an APD seen in the left-hand upper corner of frame 2(c) disappears with the formation of an approximately horizontal nc-APB seen in the frame 2(d) which moves down sweeping two adjacent cAPBs. When it reaches a triple junction of its adjacent c-APB with two other nc-APBs seen in frame 2(d), the resulting junction of three nc-APBs immediately splits into two triple junctions - that of a pair of c-APBs with an nc-APB and that of a c-APB with two nc-APBs, with the formation of a new APD seen in frame 2(e).

The splitting effect is related to very small excess energies of c-APBs in short-rangeinteraction systems, as well as to geometrical constraints on the formation of such APBs between APDs of different types. An APD of a given type (say, A) can form a c-APB of a certain orientation (say, (100)) with an APD of only one type (B) but not with APDs of two other types (C and D) [30]. Therefore, it can be energetically favourable to split an nc-APB between APDs of types A and C (or D) with the formation of an additional APD of type B if the area of a newly formed nc-APB between APDs of types B and C is lower than the area of an initial nc-APB between APDs of types A and C. Let us also note that our simulation revealed the splitting effect only for model 1 with $|\epsilon|=\left|v_{2} / v_{1}\right|=0.125$, while for models 2 and 3 with larger $|\epsilon|$ this effect was not observed. 


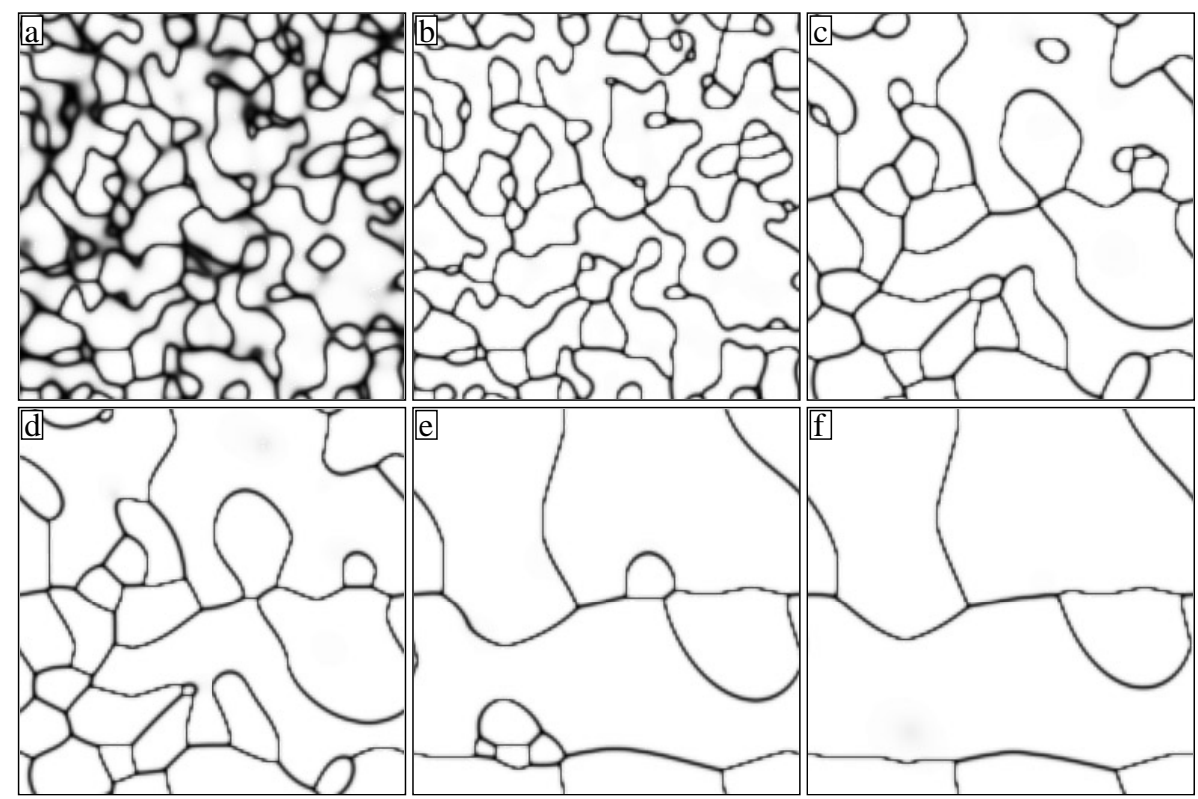

Figure 8. As figure 2, but for model 4 with $V_{b}=128^{2} \times 1$ at $T^{\prime}=1.15$ and the following values of $t^{\prime}$ : (a) 2; (b) 3; (c) 20; (d) 30; (e) 150; and (f) 200 .

Among other processes illustrated by figures 2,4 and 7 , one can note a peculiar effect of 'tearing off' of some nc-APBs. Two such events are seen in frames 7(b) and 7(c), one to the right of the centre and another one above the centre. However, this effect is not specific to short-range-interaction systems, and the lower part of frames 9(a)-9(c) shows it for model 5 with a relatively longe-range ('extended-range') interaction.

Let us discuss the effect of non-stoichiometry on the evolution. To this end we have made simulations for model 2 at the same reduced temperature $T^{\prime}=T / v_{1}=0.5$ as in the simulation shown in figure 5 but at different concentrations, $c=0.22$ and $c=0.32$. In the phase diagram for this model shown in figure 1(a) these points $c, T^{\prime}$ correspond to approximately the same distance, $\delta c \simeq 0.02$, from the two-phase field, $\mathrm{A} 1+\mathrm{L} 1_{2}$ and $\mathrm{L} 1_{2}+\mathrm{L} 1_{0}$, respectively. Some results of this simulation are shown in figures 5 and 6 . Comparison of frames 4(c), 5(a) and 5(c), as well as 4(d), 5(b) and 5(d), shows that general features of microstructures for all three compositions are similar, though at lower concentration $c=0.22$ the number of conservative APBs in microstructures is somewhat reduced while the evolution rate is slightly enhanced. However, the structure of APBs reveals significant changes with composition, which are seen in figures 5 and 6 . At lower $c=0.22$, the local concentration $\bar{c}_{i}$ and the local order parameters $\left|\eta_{\alpha i}\right|$ in the APB region are notably lower than those at stoichiometric $c=0.25$, which indicates that APBs are locally disordered. In contrast, at higher $c=0.32$ the local concentrations $\bar{c}_{i}$ and the local order parameters $\left|\eta_{\alpha i}\right|$ for some $\alpha=\alpha_{0}$ increase near the APB, while for two other $\alpha \neq \alpha_{0}$ the values $\left|\eta_{\alpha i}\right|$ decrease here. This indicates that the APB is locally $\mathrm{L} 1_{0}$ ordered. The effects of local $\mathrm{L}_{0}$ ordering or disordering of $\mathrm{APBs}$ in the $\mathrm{L}_{2}$ phase were first discussed by Kikuchi and Cahn [30] for the nearest-neighbour interaction model near the congruent point. Figure 6 displays these effects in terms of local order parameters $(24 a)$ and local concentration $(24 b)$. It also illustrates the concentrational dependence of these effects for both non-conservative APBs (at $x_{1} \simeq 16$ and $x_{1} \simeq 48$ ) and conservative APBs (at $x_{1} \simeq 58$ ). At stoichiometric $c=0.25$, some parts of APBs ('steps' or 'ledges' in figure 4) are weakly 


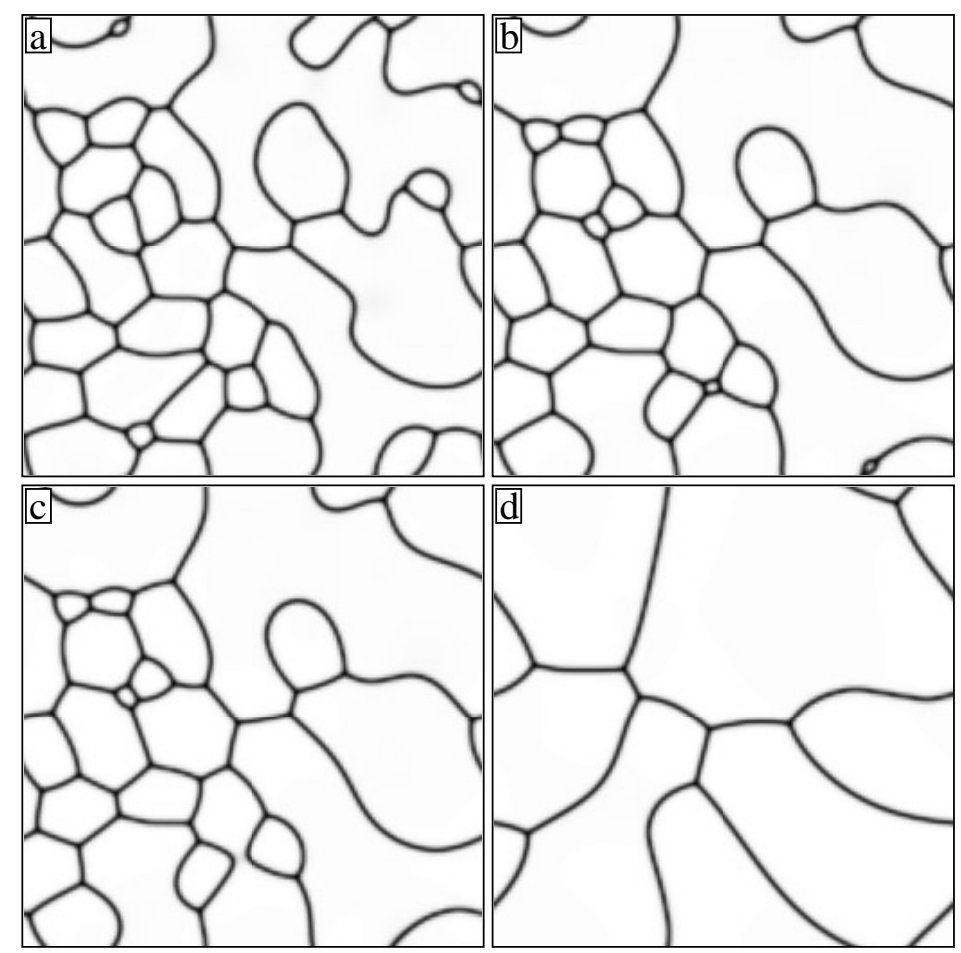

Figure 9. As figure 8, but for model 5 at $T^{\prime}=1.34$ and the following values of $t^{\prime}$ : (a) 20; (b) 44; (c) 46; and (d) 200 .

$\mathrm{L} 1_{0}$ ordered, while other parts adjacent to them are locally disordered, which is illustrated by figure 6(b). A more detailed discussion of the structure and properties of APBs in the $\mathrm{L}_{2}$ phase for various compositions, temperatures and types of interaction will be given elsewhere.

Let us now consider the evolution for the $\mathrm{Ni}$-Al-type model 4 with a relatively long-range interaction. This is illustrated by figure 8 . Frames 8 (a) and $8($ b) show an effect of thinning of APBs at the early stages of evolution due to the development of ordering; similar effects were observed for all other models. Frames 8 (b)-8(f) show that the microstructures for this system include mainly non-conservative APBs. However, the conservative APBs are present, too, and have a noticeable effect on microstructures. In particular, the evolution of ordered domains in the central part of frames $8(\mathrm{c})-8(\mathrm{e})$ as well as in the lower left-hand part of frames $8(\mathrm{~d})-8(\mathrm{f})$ illustrates the effect of transformation of non-conservative APBs into conservative ones. The final microstructure shown in frame 8(f) reveals a noticeable anisotropy in the distribution of APBs and is qualitatively similar to that observed in a real $\mathrm{Ni}_{3} \mathrm{Al}$-based alloy (see figure 6 in [26]).

Figure 9, for model 5, illustrates the evolution of a system with an 'extended-range' interaction. All APBs seen in the microstructures are non-conservative and are distributed isotropically. Triple junctions of APBs at later stages of the evolution correspond to approximately equiangle configurations with angles of about $2 \pi / 3$, which is characteristic for isotropic systems. The comparison of the microstructures in frames 8(f) and 9(d) (each including only four different ordered domains) again indicates a noticeable anisotropy in the $\mathrm{Ni}$-Al-type model 4 compared to the isotropic model 5. Let us note that the microstructure in frame 9(d) is similar to those observed for $\mathrm{Cu}-\mathrm{Pd}$ alloys (see figure 9 in [24]). 


\section{Features of evolution under $\mathrm{A} 1 \rightarrow \mathrm{A} 1+\mathrm{L1}_{2}$ transition}

In this section we consider the $\mathrm{A} 1 \rightarrow \mathrm{A} 1+\mathrm{L}_{2}$ transition. For simplicity we suppose the transformation temperature $T$ to be lower than the ordering spinodal temperature $T_{S}(c)$, so as to avoid discussing the problems of nucleation. First we consider the dependence of microstructural evolution on the type of interaction. As discussed above, for the $\mathrm{A} 1 \rightarrow \mathrm{L} 1_{2}$ transition such dependence is rather sharp due to the predominance of highly anisotropic conservative APBs in the short-range-interaction systems and the absence of anisotropy in the extended-range-interaction systems. In the two-phase region under consideration, different $\mathrm{L1}_{2}$-ordered domains are usually surrounded by the disordered phase; thus the possible anisotropy of the microstructures depends on the anisotropy of the energies of interphase boundaries (IPBs). For the nearest-neighbour interaction model, Kikuchi and Cahn [30] found the IPB energies to be only slightly anisotropic, in great contrast to the highly anisotropic APB energies. Therefore, one can expect the microstructures under $\mathrm{A} 1 \rightarrow \mathrm{A} 1+\mathrm{L}_{2}$ transition (except possibly at its early stage) to display neither significant anisotropy nor sensitivity to the interaction range as long as the latter remains finite, i.e. in the absence of the significant elastic forces discussed below.

Figure 10 illustrates the evolution for model 1 with a short-range interaction. Frame 10(a) (which may be compared with frame 2(b)) shows the stage of initial congruent ordering (mentioned in section 1 and discussed below) when the newly formed APBs between different ordered domains begin to be wetted by the disordered phase. In this frame one can recognize many (100)-oriented IPBs obtained by wetting of the initial conservative APBs. Frames

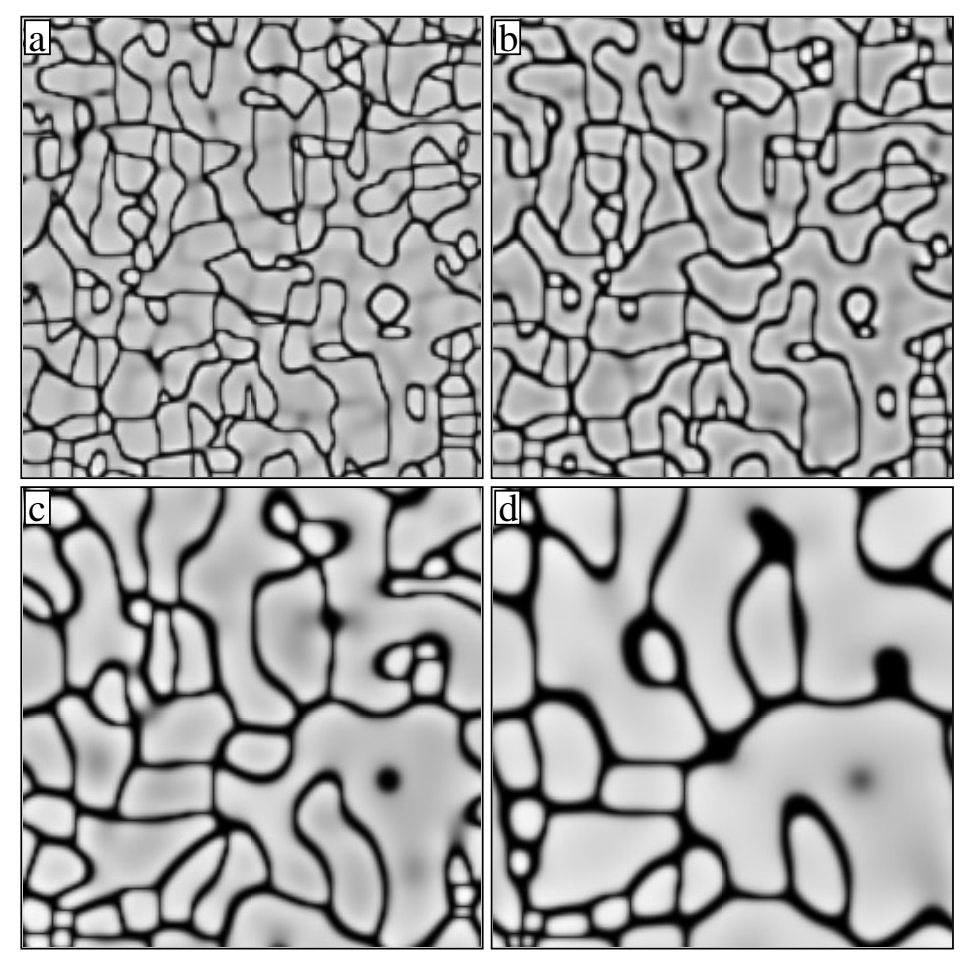

Figure 10. As figure 2, but for the $\mathrm{A} 1 \rightarrow \mathrm{A} 1+\mathrm{L} 1_{2}$ transformation with $V_{b}=128^{2} \times 1$ at $c=0.17$, $T^{\prime}=0.24 \mathrm{~K}$ and the following values of $t^{\prime}$ : (a) 3 ; (b) 10 ; (c) 100 ; and (d) 300 . 
10(b)-10(d) show that after the transformation of these APBs into IPBs is completed, their initial anisotropy falls off, in agreement with the above-mentioned conclusions [30] about a weak anisotropy of IPB energies. In the microstructure of frame 10(d) the anisotropy is almost absent, in great contrast with the case for frame 2(f) for the same model and the same evolution time but for the $\mathrm{A} 1 \rightarrow \mathrm{L}_{2}$ transition. As expected, the simulations for models 2, 3, 4 and 5 show still lower anisotropy of microstructures, which can be illustrated by frame 12(a) for early $t^{\prime}=10$ (when the elastic interaction $v^{e l}$ that distinguishes between the models $4^{\prime}$ and 4 is still insignificant; see below). Therefore, the type of interaction affects microstructures only at early stages of the transformation corresponding to congruent ordering.

Let us consider the effects of elastic forces. Their influence on the microstructural evolution under alloy decomposition with ordering was thoroughly discussed by Khachaturyan and co-workers with the use of phenomenological kinetic equations; see e.g. [4-6]. In particular, a recent work [6] describes these effects under $\mathrm{A} 1 \rightarrow \mathrm{A} 1+\mathrm{L}_{2}$ transformation for a phenomenological model fitted to the $\mathrm{Ni}-\mathrm{Al}$ alloys. The microscopical treatment of these problems described below can complement and verify the phenomenological approaches.

We have made simulations for models $2^{\prime}$ and $4^{\prime}$ described in section 3 . The elastic interaction $v^{e l}$ for these models corresponds to Ni-enriched $\mathrm{Ni}-\mathrm{Al}$ alloys. Thus the values $v_{n}^{e l}$ for several first neighbours $n$ can be estimated from the calculations [37] for the Ni-based alloys: $v_{1} \simeq-130 \mathrm{~K}, v_{2} \simeq-36 \mathrm{~K}, v_{3} \simeq 6 \mathrm{~K}$ and $v_{4} \simeq 19 \mathrm{~K}$. The 'chemical' contributions $v_{n}^{c}$ to the total $v_{n}=v_{n}^{c}+v_{n}^{e l}$ for the models $2^{\prime}$ and $4^{\prime}$ are given in section 3 and they exceed the $v_{n}^{e l}$ by an order of magnitude. Hence the phase diagrams for models $2^{\prime}$ and $4^{\prime}$ virtually coincide with those for models 2 and 4 presented in figure 1 . However, as discussed by Khachaturyan and co-workers [4], the influence of $v^{e l}$ on the microstructures increases with the characteristic size $l$ of the precipitates, and at sufficiently large $l$ it results in the alignment of IPBs along the elastically soft (100) directions. Khachaturyan and co-workers mentioned that the degree of this alignment depends on the elastic anisotropy energy $v_{a n}^{e l}$ which can be estimated as the difference between the asymptotic values $v_{a s}^{e l}$ of the Fourier components $v^{e l}(\boldsymbol{k})$ at $|\boldsymbol{k}| \rightarrow 0$ for the elastically stiff and elastically soft directions, (110) and (100):

$$
v_{a n}^{e l}=v_{a s}^{e l}(110)-v_{a s}^{e l}(100) \text {. }
$$

The characteristic precipitate size $l_{c}$ for which the elastic anisotropy effects become noticeable depends on the ratio of $v_{a n}^{e l}$ to the characteristic IPB energy (which for estimates can be taken as the critical temperature of ordering, $T_{c}$ ), as well as on the ratio of $v_{a n}^{e l}$ to the transformation temperature $T$.

The results of our simulation presented in figures 11 and 12 can be used to illustrate these considerations and make them specific. For the Ni-Al-type model of elastic interaction employed, the $v_{a n}^{e l}$-value (which can be found using the expression for $v_{a s}^{e l}$ given in equation (29) of [18]) is about $700 \mathrm{~K}$. Therefore, the ratios $\lambda_{c}=v_{a n}^{e l} / T_{c}$ and $\lambda_{T}=v_{a n}^{e l} / T$ for model $2^{\prime}$ are about 0.9 and 1.75 , respectively, while for model $4^{\prime}$ they are much lower: $\lambda_{c}\left(4^{\prime}\right) \simeq 0.35$ and $\lambda_{T}\left(4^{\prime}\right) \simeq 0.7$. Thus the elastic anisotropy effects for model $2^{\prime}$ are much stronger than those for model $4^{\prime}$. In addition, the chemical interactions $v_{n}^{c}$ for model $2^{\prime}$ are short range, which leads to a notable (100) alignment of APBs under congruent ordering. Unlike in the above-discussed case of no elastic interaction, this initial anisotropy is not lost after the transformation of these APBs into IPBs, but is fixed and enhanced by significant elastic forces. All of these factors result in a noticeable alignment of the precipitates even at moderate sizes $l_{c} \sim 10 a$.

Figure 11 also illustrates a number of other effects of elastic interaction noted by Khachaturyan and co-workers. In particular, a suppression of the coalescence of differently ordered precipitates discussed in [5,6] is seen in the left-hand lower part of frames 11(c)-11(f), as well as in frame 11(c) for a number of pairs of adjacent precipitates: those with centres at 


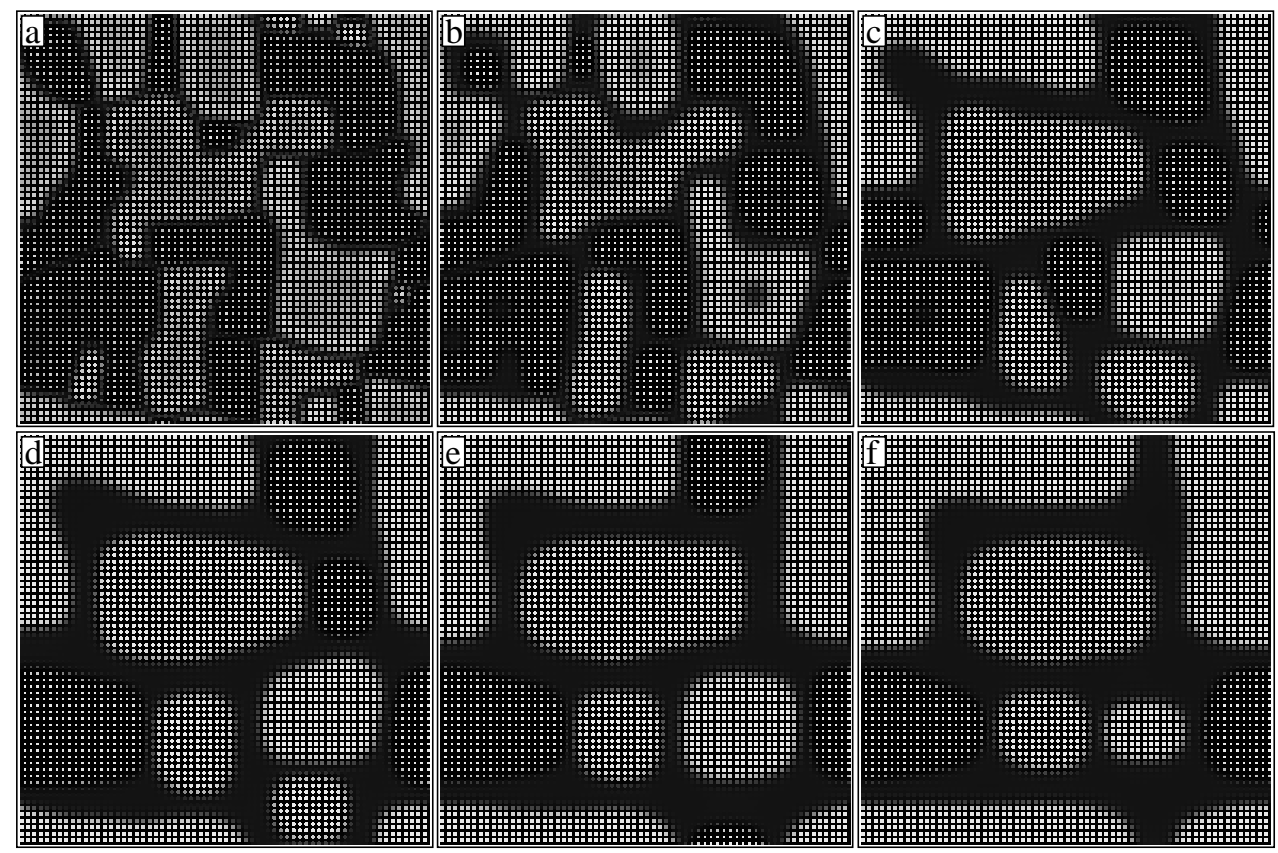

Figure 11. As figure 10, but with $V_{b}=64^{2} \times 1$ shown in the $c$-representation for model $2^{\prime}$ at $c=0.17, T=400 \mathrm{~K}$ and the following values of $t^{\prime}$ : (a) 10; (b) 100; (c) 500; (d) 1000; (e) 2000; and (f) 4200 . The grey level varies linearly with $c_{i}$ between its minimum and maximum values from completely dark to completely bright.

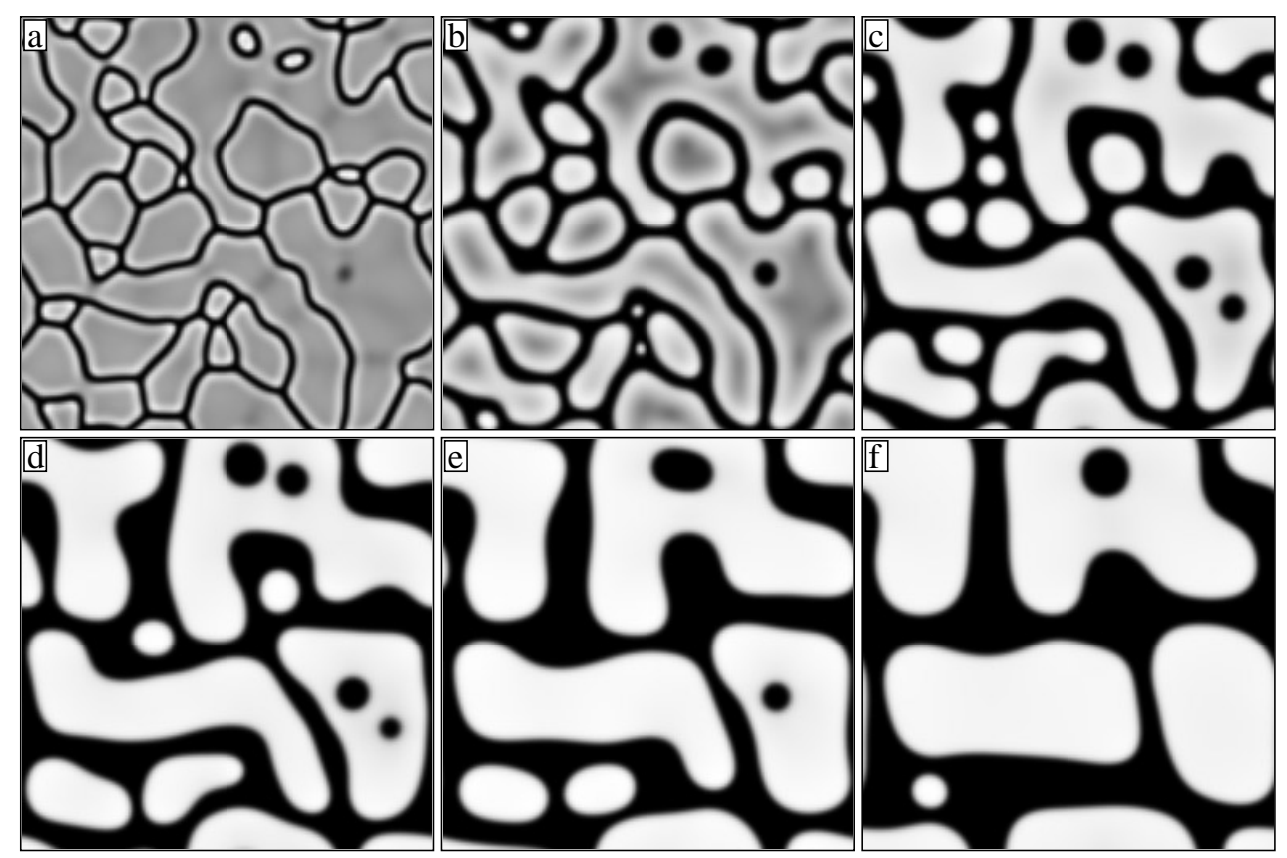

Figure 12. As figure 10, but for the Ni-Al-type model $4^{\prime}$ at $c=0.17, T=1000 \mathrm{~K}$ and the following values of $t^{\prime}$ : (a) 10; (b) 100; (c) 500; (d) 1000; (e) 2000; and (f) 4000 . 
$\left(x_{1} \simeq 10, x_{2} \simeq 18\right)$ and $\left(x_{1} \simeq 5, x_{2} \simeq 33\right) ;$ at $\left(x_{1} \simeq 48, x_{2} \simeq 23\right)$ and $\left(x_{1} \simeq 32, x_{2} \simeq 25\right)$; at $\left(x_{1} \simeq 48, x_{2} \simeq 23\right)$ and $\left(x_{1} \simeq 44, x_{2} \simeq 7\right)$; etc. In the course of further evolution, the smaller precipitate of each pair usually dissolves ('evaporates') but never coalesces with a differently ordered neighbour. Frame 11(f) also shows such microstructural features as a 'rectangleshaped' precipitate (in the left-hand upper corner) and a 'discontinuous rafting' of chains of approximately rectangular particles. These features were also discussed by Khachaturyan and co-workers and they agree well with experimental observations $[5,6]$.

Figure 12 illustrates a different type of evolution. It corresponds to the Ni-Al-type model $4^{\prime}$ for which the influence of elastic anisotropy on microstructures (characterized by the abovementioned parameters $\lambda_{c}$ and $\lambda_{T}$ ) is much weaker than that for model $2^{\prime}$, while the chemical interactions $v_{n}^{c}$ are basically extended-range ones. Because of that, the congruent ordering stage here reveals no anisotropy of APBs, and the IPBs formed from these APBs and shown in frame 12(a) are isotropic too. During the next stage of the transformation, both concentration and order parameters within the ordered precipitates increase, tending to their equilibrium values in the $\mathrm{L}_{2}$ phase (at the boundary of the single-phase region in the $c, T$-plane). This process leads to the 'shrinking' of the precipitates - a decrease of their volume due to the atom number conservation, which can result, in particular, in the formation of 'holes', i.e. islands of the disordered phase within some extended precipitates. This stage is illustrated by frames 12(b) and 12(c), and these microstructures still do not show the effects of elastic anisotropy. These effects begin to be noticeable only at the next, coarsening stage when the elastically misfitted ordered and disordered regions become sufficiently large. It is illustrated by frames 12(d)-12(f) and corresponds to characteristic sizes $l_{c} \gtrsim 50 a$. With a further development of coarsening, the manifestations of elastic effects in microstructures become more notable. However, for all stages of evolution considered they remain much less pronounced than those shown in figure 11 for model $2^{\prime}$.

Let us now discuss the problem of congruent ordering under the $\mathrm{A} 1 \rightarrow \mathrm{A} 1+\mathrm{L}_{2}$ transformation. As mentioned in section 1, the presence of such an initial transient stage under alloy decomposition with ordering follows from rather general physical arguments and it was supported by experimental observations for a number of alloy systems [4, 29, 33]. However, in the HREM study of the early decomposition stage of Al-Li alloys, Haasen and co-workers [27] observed microstructures seeming to be incompatible with the occurrence of congruent ordering. In these microstructures, many neighbouring ordered precipitates (separated by a disordered layer) are 'in-phase', i.e. belong to the same type of $\mathrm{L}_{2}$-ordered domains. At the same time, the congruent ordering corresponds to the microstructure of differently ordered neighbouring domains separated by APBs which later on transform into IPBs; see e.g. frames 10(a), 11(a) and 12(a). Haasen and co-workers concluded that these observations contradict the occurrence of congruent ordering under the $\mathrm{A} 1 \rightarrow \mathrm{A} 1+\mathrm{L} 1_{2}$ transition in the Al-Li system.

To clarify the problem, we made simulations of the $\mathrm{A} 1 \rightarrow \mathrm{A} 1+\mathrm{L}_{2}$ transition under conditions similar to those of experiments by Haasen and co-workers, i.e. at $c, T$-points positioned in the $c, T$-plane not far from the ordering spinodal. For such $c, T$-values the initial ordered domains formed under congruent ordering are sufficiently large and are similar to those observed by Haasen and co-workers. Some results of our simulations are shown in figures 13-15. The upper frames in figures 13 and 14 correspond to 3D simulation and are shown in a $c$-representation to facilitate comparison with the HREM images in [27]. The lower frames in these figures and figure 15 correspond to 2D simulations and show more sizable microstructures for which it is easier to follow the evolution in an $\eta^{2}$-representation.

In all of our simulations we observed the stage of congruent ordering which is seen, for example, in frames 13(c), 13(e), 14(b), 14(d) and 15(a). However, both before and after this 


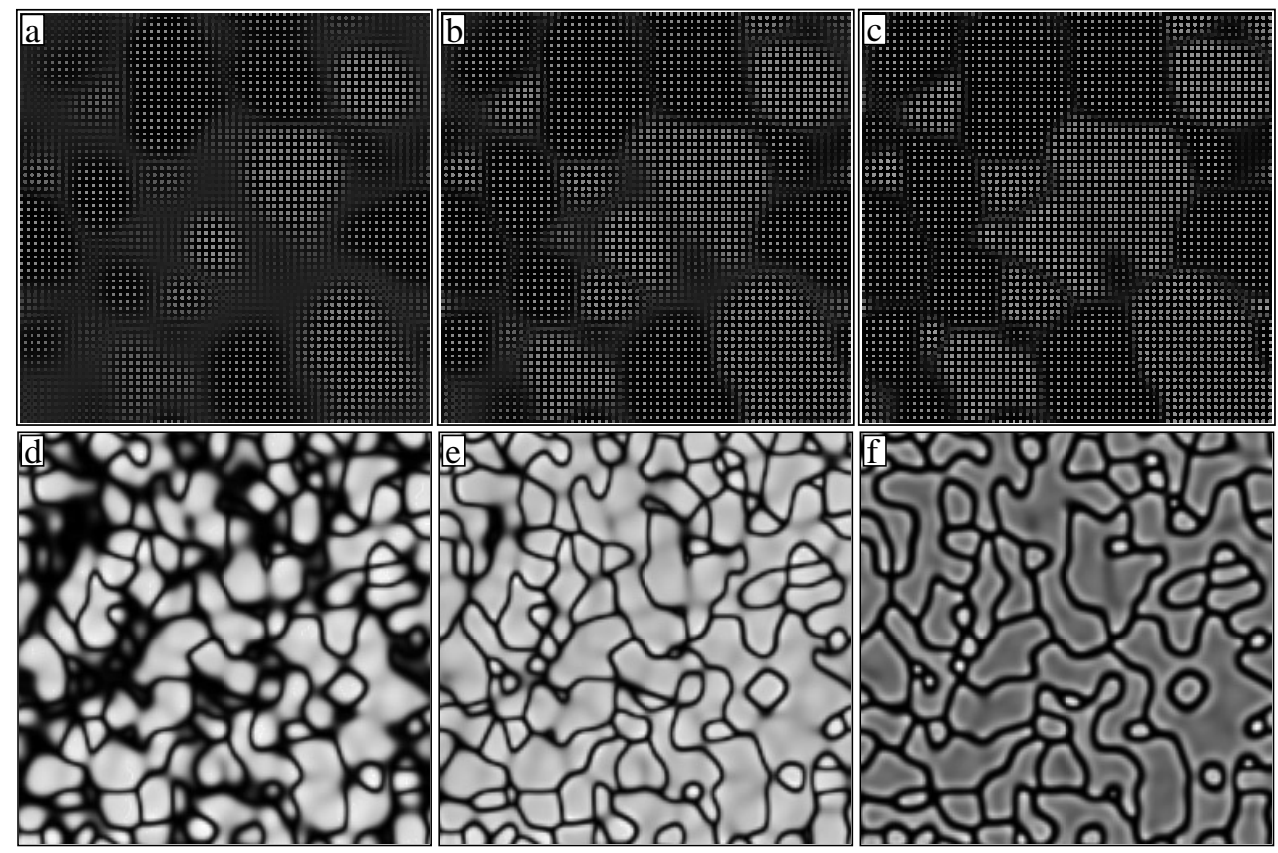

Figure 13. As figure 11, but for model 2 at $c=0.13, T^{\prime}=0.3$. The three upper frames correspond to 3D simulation with $V_{b}=60^{3}$ shown in the $c$-representation at $x_{3}=0$ and the following values of $t^{\prime}$ : (a) 3.2 ; (b) 3.5 ; and (c) 4 . The three lower frames correspond to 2D simulation with $V_{b}=128^{2} \times 1$ shown in the $\eta^{2}$-representation at the following values of $t^{\prime}$ : (d) 2.6 ; (e) 3 ; and (f) 10 .

stage we observed a number of microstructures with neighbouring in-phase domains separated by a disordered layer.

Figures 13-15 illustrate at least three possible mechanisms for the formation of neighbouring in-phase domains. The first one is typical of a stage before the completion of congruent ordering and corresponds to the coalescence of neighbouring in-phase domains being initially approximately equiaxial, with the formation of more extended irregularly shaped domains. Such processes can be observed, for example: in frames 13(a), 13(b) near the points $\left(x_{1} \simeq x_{2} \simeq 30\right),\left(x_{1} \simeq x_{2} \simeq 7\right)$ and $\left(x_{1} \simeq 13, x_{2} \simeq 26\right)$; in the upper left-hand and lower righthand part of frames 14(a), 14(b); and for many neighbouring domains in frames 13(d) and 14(c) which subsequently coalesce, with the formation of extended domains seen in frames 13(e) and 14(d). Let us note that the microstructures in frames 13(a), 13(b) and 14(a) illustrating this process are quite similar to those observed by Haasen and co-workers [27].

The second mechanism is observed after the completion of congruent ordering and corresponds to the disappearance of a small 'out-of-phase' domain positioned between larger in-phase domains. It is seen, for example, in the left-hand lower part of frame 13(e) where a small domain at $x_{1} \simeq 12, x_{2} \simeq 15$ separates two pairs of larger domains which are in phase with each other (which can be seen in the $c$-representation). After the disappearance of this small domain shown in frame 13(f) these two pairs of in-phase domains become neighbours. Such a mechanism is also seen in the left-hand part of frames 10(b) and 10(c), at $x_{1} \simeq 30$ and $x_{2}$ between about 60 and 80, where the disappearance of several small domains results in a microstructure with several in-phase neighbours. This mechanism is not so typical as the first one but we observed it in many simulations too. 

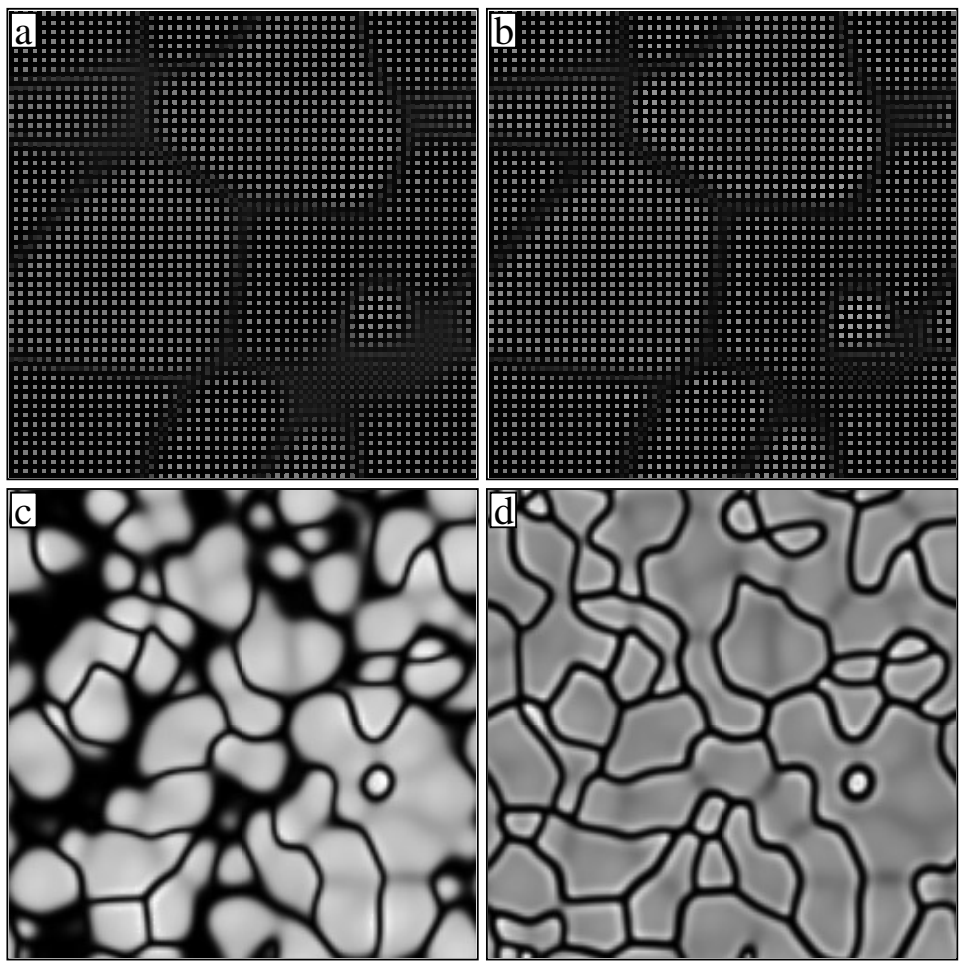

Figure 14. As figure 13, but at $c=0.12$. The two upper frames correspond to $3 \mathrm{D}$ simulation with $V_{b}=50^{3}$ shown in the $c$-representation at $x_{3}=0$ and the following values of $t^{\prime}$ : (a) 8.6; and (b) 10. The two lower frames correspond to $2 \mathrm{D}$ simulation with $V_{b}=128^{2} \times 1$ shown in the $\eta^{2}$-representation at the following values of $t^{\prime}$ : (c) 5.6 ; and (d) 8 .

The third mechanism of the formation of neighbouring in-phase domains is illustrated in figure 15. It corresponds to a later stage of transformation when a 'shrinking' of initially extended ordered domains (mentioned above in the discussion of frames 12(a)-12(c)) results in a tearing-off of some of them. Comparison of frames 15(a)-15(d) shows that many ordered precipitates in frames 15(c) and 15(d) have in-phase neighbours formed in this way. This mechanism was observed only in the simulations with $c, T$-values that were quite close to the ordering spinodal $c_{s}(T)$ (for example, at $c-c_{s} \simeq 0.01$ for the simulation shown in figure 15), and the microstructures corresponding to this mechanism do not seem to resemble those seen by Haasen and co-workers [27]. However, it can illustrate one more opportunity for the formation of neighbouring in-phase domains.

Finally, let us discuss the temporal evolution of local concentrations and local order parameters in the course of congruent ordering. Speaking formally, the term 'congruent' implies local concentrations to be unchanged and equal to the initial constant concentration $c$ (saying nothing about the small initial fluctuations $\delta c_{i}$ ) while the order parameter grows and approaches its equilibrium value for the given $c$. However, as was repeatedly noted in this and previous sections, the local concentration within the APBs at sub-stoichiometric compositions is depleted even in the homogeneous $\mathrm{L}_{2}$ phase, while in the two-phase $\mathrm{A} 1+\mathrm{L}_{2}$ region the initially formed APBs are wetted by the disordered phase which results in a further depletion of local concentrations. Therefore, it is not clear a priori whether APBs being formed under congruent ordering have time to equilibrate locally and deplete their local concentration, or 

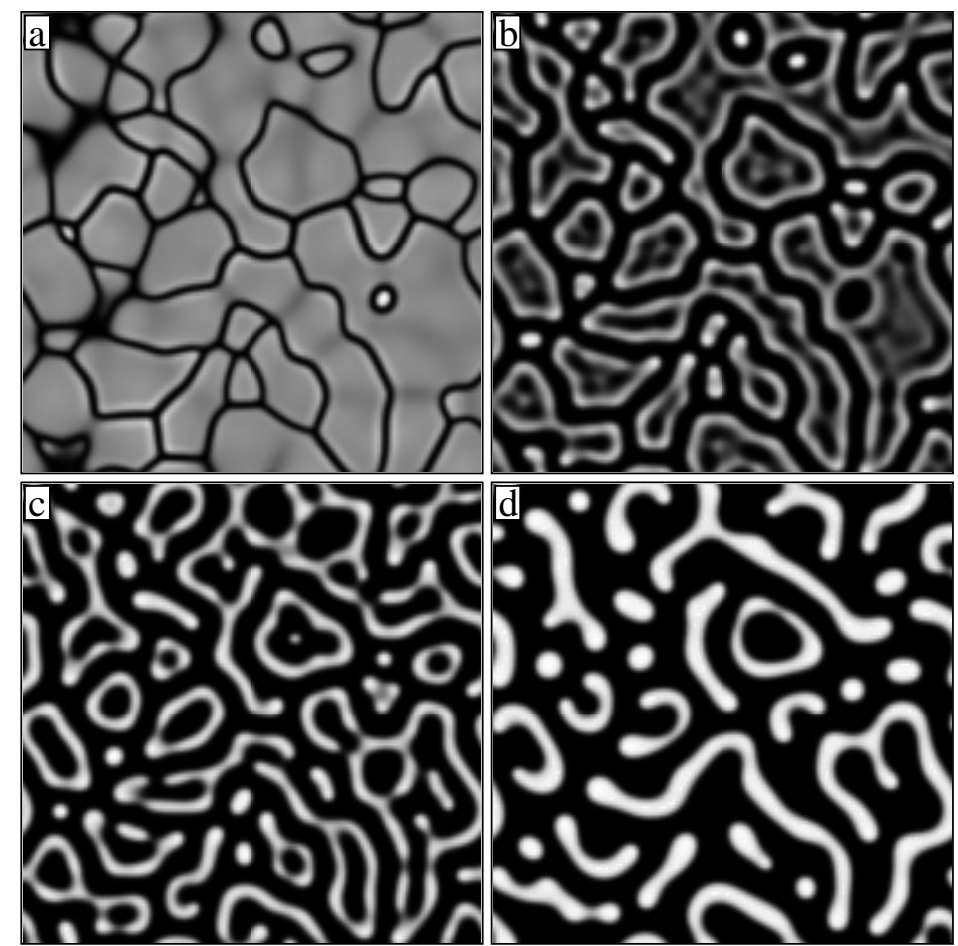

Figure 15. As figure 13, but for 2D simulation with $V_{b}=128^{2} \times 1$ for model 3 at $c=0.11$, $T^{\prime}=0.5$ shown in the $\eta^{2}$-representation at the following values of $t^{\prime}$ : (a) 7; (b) 40; (c) 100 ; and (d) 350 .

whether for some time interval this local concentration preserves the initial constant value $c$. Note that such depletion of local concentrations within APBs requires only local atom exchanges just like the congruent ordering [33]. Therefore, generally speaking, the time intervals needed for the formation of congruently ordered domains and for the depletion of local concentrations within the APBs separating these domains should be similar.

To study this point in more detail, we analysed the results of our simulation as follows. We divide all lattice sites into locally ordered and locally disordered ones. The degree of local ordering is characterized by the value $\eta_{i}^{2}=\eta_{1 i}^{2}+\eta_{2 i}^{2}+\eta_{3 i}^{2}$, and at each evolution time $t$ we determine the mean value $\bar{\eta}^{2}(t)$, averaging the $\eta_{i}^{2}$-values over all lattice sites. Then we classify (somewhat arbitrarily) the sites with $\eta_{i}^{2}>0.5 \bar{\eta}^{2}(t)$ as locally ordered and those with $\eta_{i}^{2}<0.5 \bar{\eta}^{2}(t)$ as locally disordered. After that we find the average concentration and the average squared order parameter for locally ordered and locally disordered regions separately:

$$
\begin{aligned}
c_{+} & =\frac{1}{N_{+}} \sum_{i=i_{+}} c_{i} & c_{-} & =\frac{1}{N_{-}} \sum_{i=i_{-}} c_{i} \\
\eta_{+}^{2} & =\frac{1}{N_{+}} \sum_{i=i_{+}} \eta_{i}^{2} & \eta_{-}^{2} & =\frac{1}{N_{-}} \sum_{i=i_{-}} \eta_{i}^{2}
\end{aligned}
$$

where the subscript ' + ' or ' - ' corresponds to locally ordered or locally disordered sites $i_{+}$ or $i_{-}$while $N_{+}$or $N_{-}$means the total number of such sites. Locally ordered regions under congruent ordering evidently correspond to ordered domains, while locally disordered regions correspond to APBs. Therefore, an analysis of the temporal evolution of $c_{ \pm}(t)$ and $\eta_{ \pm}^{2}(t)$ can 
provide information about the evolution of local concentrations and order parameters both in ordered domains and near the APBs.

This temporal evolution is illustrated in figure 16, which corresponds to the 3D simulation shown in the upper frames of figure 13. The results of other simulations are similar. Comparison of curves $c_{-}(t)$ and $\eta_{+}^{2}(t)$ in figure 16 shows that the depletion of local concentrations within APBs begins almost simultaneously with the appearance of a noticeable ordering. In other words, a newly formed APB begins to equilibrate and deplete its local concentration immediately. One can notice just a short time interval, $2.5 \lesssim t^{\prime} \lesssim 2.8$, when the rise of $\eta_{+}^{2}(t)$ notably surpasses the decrease of $c_{-}(t)$; thus within this interval the evolution may be termed almost 'truly congruent'. However, after the completion of congruent ordering in the commonly used sense of this term (which corresponds to $t^{\prime} \gtrsim 5$ in figure 16), the local concentration within APBs is already depleted. Let us also mention that the presence of such depletion of local concentrations at APBs may lead to some terminological misunderstandingin particular, in the interpretation of the results of Monte Carlo simulations $[1,2]$ concerning the occurrence of congruent ordering.

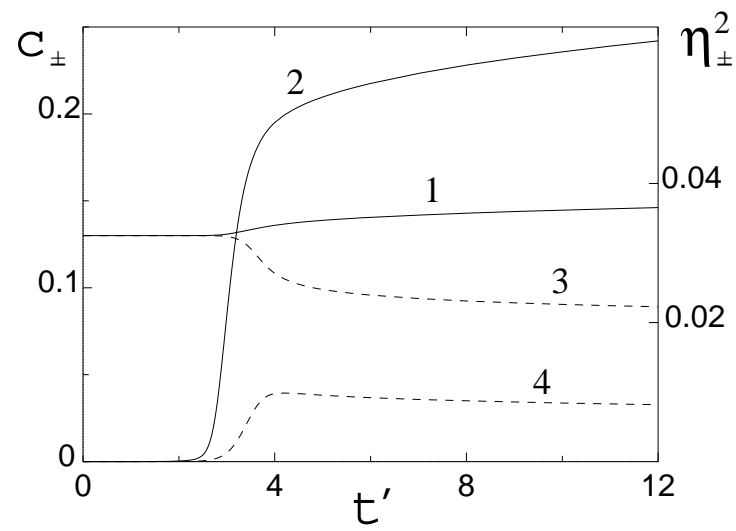

Figure 16. Temporal evolution of the mean concentrations $\bar{c}_{ \pm}$(left-hand scale) and squared order parameters $\eta_{ \pm}^{2}$ (right-hand scale) averaged over locally ordered or locally disordered regions as described in the text for the simulation shown in frames 13(a)-13(c). Curves 1,2, 3 and 4 correspond to $c_{+}, \eta_{+}^{2}, c_{-}$and $\eta_{-}^{2}$, respectively, where the subscript ' + ' or ' - ' corresponds to the locally ordered or the locally disordered region.

\section{Conclusions}

Let us summarize the main results of this work. We apply the earlier-described kinetic cluster-field approach to study the ordering kinetics in FCC alloys. First we describe the kinetic tetrahedron cluster-field method (KTCFM) which generalizes the tetrahedron clusterfield method (TCFM) used for equilibrium systems to the case of non-equilibrium and inhomogeneous alloys. We use the KTCFM to derive the kinetic equations for mean lattice site occupations which determine local concentrations and local order parameters in an alloy. These equations are sufficiently simple and convenient for numerical solution. As the TCFM describes the phase diagrams with $\mathrm{L}_{2}$ and $\mathrm{L} 1_{0}$ orderings for a number of realistic alloy models rather accurately [23], one may expect the KTCFM to describe the kinetics of such transitions with a similar accuracy.

Then we use the KTCFM to simulate the $\mathrm{A} 1 \rightarrow \mathrm{L}_{2}$ and $\mathrm{A} 1 \rightarrow \mathrm{A} 1+\mathrm{L}_{2}$ phase transformations after a quench of an alloy from the disordered A1 phase to the single-phase 
$\mathrm{L}_{2}$ field or the two-phase $\mathrm{A} 1+\mathrm{L}_{2}$ field of the phase diagram. In these simulations we employ five alloy models with different types of interaction, from the short-range-interaction model 1 to the extended-range-interaction model 5. To discuss the influence of elastic forces on the $\mathrm{A} 1 \rightarrow \mathrm{A} 1+\mathrm{L} 1_{2}$ transition, we also consider two more models, $2^{\prime}$ and $4^{\prime}$, which include the elastic interaction $v^{e l}$ with the parameters corresponding to $\mathrm{Ni}-\mathrm{Al}$ alloys. We employed both 2D and 3D simulations, and all significant features of evolution in both types of simulation were found to be similar.

Simulations of the $\mathrm{A} 1 \rightarrow \mathrm{L}_{2}$ transformation were made at stoichiometric concentration $c=0.25$ for the five above-mentioned alloy models. Our results show that the microstructural evolution sharply depends on the interaction type, particularly on the interaction range $R_{\text {int }}$. For the short-range-interaction systems, particularly for model 1 with the smallest $R_{\text {int }}$, transient microstructures include mainly the conservative antiphase boundaries (APBs) with (100)-type orientation. The distribution of APBs in such systems reveals a number of peculiar features discussed in section 3: characteristic 'step-like' APBs with (100)-oriented steps and small ledges normal to them; triple junctions of two conservative APBs normal to each other with a non-conservative APB; analogous 'quadruple' junctions; loop-like configurations of nonconservative APBs adjacent to conservative ones; etc. These features agree well with the experimental observations for $\mathrm{Cu}_{3} \mathrm{Au}$ alloy $[24,25]$. In the course of the microstructural evolution the conservative APBs remain virtually immobile, and the evolution is realized via motion of non-conservative APBs and their interaction with the conservative APBs. This interaction includes a number of specific kinetic processes: 'sweeping' of conservative APBs by a moving non-conservative APB; wetting of conservative APBs by non-conservative APBs; motion of triple junctions of two non-conservative APBs with a conservative APB along the direction of the latter; and a peculiar process of 'splitting' of a non-conservative APB into a conservative and a non-conservative APB with the formation of a triple junction or a new antiphase domain. The splitting effect is related to very small energies of the conservative APBs in short-range-interaction systems, and it was observed only in model 1 with the shortest interaction range.

We also studied the influence of non-stoichiometry on the evolution by simulating the $\mathrm{A} 1 \rightarrow \mathrm{L}_{2}$ transformation for the second-neighbour interaction model 2 at $c=0.22$ and $c=0.32$. The APB distribution does not reveal a great sensitivity to the concentration, but the internal structure of APBs shows significant compositional changes. The degree of local disordering of APBs at lower $c=0.22$ is notably higher than at $c=0.25$, while at $c=0.32$ both the non-conservative and conservative APBs show a significant degree of local ordering of the $\mathrm{L} 1_{0}$ type.

The microstructural evolution for the fourth-neighbour-interaction models 4 and 5 greatly differs from that for the short-range interaction systems. Transient microstructures in model 4 (which corresponds to a Ni-Al-type alloy) include mainly the non-conservative APBs, and the APB distribution reveals only a slight anisotropy. However, the conservative APBs are also present here and have a noticeable effect on the microstructures. The latter agrees with the experimental observations for $\mathrm{Ni}_{3} \mathrm{Al}$-type alloys [26]. At the same time, the microstructures for the extended-range-interaction model 5 show neither conservative APBs nor anisotropy in the APB distribution, while the triple junctions of APBs form approximately equiangle configurations characteristic of isotropic systems. These microstructures are quite similar to those observed in CuPd alloys [24].

Simulations of the $\mathrm{A} 1 \rightarrow \mathrm{A} 1+\mathrm{L}_{2}$ transition show that in the absence of significant elastic interaction the microstructural evolution depends on the interaction type much more weakly than that in the case of the $\mathrm{A} 1 \rightarrow \mathrm{L}_{2}$ transition. For both short- and extendedrange-interaction systems, the interphase boundary (IPB) energies are approximately isotropic, 
greatly at variance with the APB energies in the $\mathrm{L}_{2}$ phase which are highly anisotropic in the short-range-interaction systems and virtually isotropic in the extended-range-interaction systems. Because of this, the transient microstructures under the $\mathrm{A} 1 \rightarrow \mathrm{A} 1+\mathrm{L}_{2}$ transition in the systems with short-range interaction can show anisotropy only at the first stages of evolution, when APBs formed under congruent ordering are not yet strongly wetted by the disordered phase. Later on, this wetting transforms the APBs into IPBs and the initial anisotropy falls off.

The effect of elastic forces on microstructural evolution was studied in the simulations for models $2^{\prime}$ and $4^{\prime}$ with short-range and extended-range chemical interactions, respectively. The results of these simulations agree with the phenomenological description of elastic effects developed by Khachaturyan and co-workers [4-6], and our simulations illustrate and make specific many points noted by these authors. In particular, for model $2^{\prime}$ the elastic effects are manifested even at early stages of the transformation, shortly after the completion of congruent ordering when the sizes of ordered precipitates are relatively small; this is related to the shortrange character of the chemical interactions. In contrast, for the Ni-Al-type model $4^{\prime}$ these effects become noticeable only at the advanced stages of coarsening, when the precipitate sizes $l$ become sufficiently large: $l \gtrsim 50 a$.

We also discuss the problem of transient congruent ordering mentioned in section 1 . The presence of this stage under the $\mathrm{A} 1 \rightarrow \mathrm{A} 1+\mathrm{L}_{2}$ transition was recently questioned as, at the early stages of this transition in Al-Li alloys, Haasen and co-workers [27] observed microstructures with neighbouring in-phase-ordered domains separated by a disordered layer, which seemed to be incompatible with an occurrence of congruent ordering. To clarify the problem, we made simulations of the $\mathrm{A} 1 \rightarrow \mathrm{A} 1+\mathrm{L}_{2}$ transition under conditions similar to those of the experiments of Haasen and co-workers. In all of our simulations, we observed the stage of congruent ordering. At the same time, both before and after this stage we observed many microstructures with neighbouring in-phase domains, and some of the simulated microstructures are quite similar to those observed by Haasen and co-workers [27]. Therefore, these experiments can correspond to other stages of evolution and do not contradict the assertion of the occurrence of congruent ordering. We also discuss the temporal evolution of local concentrations and local order parameters in the course of congruent ordering. We show that a depletion of local concentration near APBs begins virtually simultaneously with the appearance of ordered domains, and upon the completion of congruent ordering the local concentration within the APB is already depleted. Therefore, the commonly used term 'congruent ordering' actually corresponds to the presence of APBs with locally equilibrated, depleted concentration rather than to a 'strictly congruent' state with an unchanged initial concentration throughout the alloy.

\section{Acknowledgments}

The authors are much indebted to Georges Martin for numerous stimulating discussions. This work was supported by the Russian Fund of Basic Research, Grant No 97-02-17842.

\section{References}

[1] Abinandanan T A, Haider F and Martin G 1994 Solid-Solid Phase Transformations ed W C Johnson et al (Warrendale, PA: Minerals, Metals and Materials Society) p 443

[2] Okuda H and Osamura K 1994 Acta Metall. Mater. 421337

[3] Frontera C, Vives E, Castan T and Planes A 1997 Phys. Rev. B 55212

[4] Chen L-Q, Wang Y Z and Khachaturyan A G 1994 Statics and Dynamics of Alloy Phase Transformations (NATO Advanced Study Institute, Series B: Physics, vol 319) ed A Gonis and P E A Turchi (New York: Plenum) p 587 
[5] Wang Y, Chen L-Q and Khachaturyan A G 1994 Solid-Solid Phase Transformations ed W C Johnson et al (Warrendale, PA: Minerals, Metals and Materials Society) p 245

[6] Wang Y, Banerjee D, Su C C and Khachaturyan A G 1998 Acta Mater. 462983

[7] Vaks V G, Beiden S V and Dobretsov V Yu 1995 Pis. Zh. Eksp. Teor. Fiz. 6165 (Engl. Transl. 1995 JETP Lett. 61 68)

[8] Dobretsov V Yu, Vaks V G and Martin G 1996 Phys. Rev. B 543227

[9] Martin G 1990 Phys. Rev. B 412279

[10] Gouyet J F 1993 Europhys. Lett. 21335

[11] Vaks V G and Beiden S V 1994 Zh. Eksp. Teor. Fiz. 1051017 (Engl. Transl. 1994 Sov. Phys.-JETP 78 546)

[12] Dobretsov V Yu, Martin G, Soisson F and Vaks V G 1995 Europhys. Lett. 31417

[13] Vaks V G 1996 Pis. Zh. Eksp. Teor. Fiz. 63447 (Engl. Transl. 1996 JETP Lett. 63 471)

[14] Belashchenko K D and Vaks V G 1997 Zh. Eksp. Teor. Fiz. 112714 (Engl. Transl. 1997 Sov. Phys._JETP 85 390)

[15] Dobretsov V Yu and Vaks V G 1998 J. Phys.: Condens. Matter 10226 Dobretsov V Yu and Vaks V G 1998 J. Phys.: Condens. Matter 102275

[16] Belashchenko K D and Vaks V G 1998 J. Phys.: Condens. Matter 101965

[17] Nastar M, Martin G and Dobretsov V Yu 1999 Phil. Mag. A at press

[18] Belashchenko K D, Samolyuk G D and Vaks V G 1999 J. Phys.: Condens. Matter 1110567

[19] Kikuchi R 1951 Phys. Rev. 81988

[20] Mohri T, Sanchez J M and de Fontaine D 1985 Acta Metall. 331171

[21] Finel A 1994 Statics and Dynamics of Alloy Phase Transformations (NATO Advanced Study Institute, Series B: Physics 319) ed A Gonis and P E A Turchi (New York: Plenum) p 495

[22] Vaks V G, Zein N E and Kamyshenko V V 1988 J. Phys. F: Met. Phys. 181641

[23] Vaks V G and Samolyuk G D 1999 Zh. Eksp. Teor. Fiz. 115158 (Engl. Transl. 1999 Sov. Phys.-JETP 88 89)

[24] Loiseau A, Ricolleau C, Potez L and Ducastelle F 1994 Solid-Solid Phase Transformations ed W C Johnson et al (Warrendale, PA: Minerals, Metals and Materials Society) p 385

[25] Potez L and Loiseau A 1994 J. Interface Sci. 291

[26] Cahn R W, Siemers P A and Hall E L 1987 Acta Metall. 352753

[27] Schmitz G, Hono K and Haasen P 1994 Acta Metall. Mater. 42201

[28] Okuda H, Vezin V, Osamura K and Amemiya Y 1994 Solid-Solid Phase Transformations ed W C Johnson et al (Warrendale, PA: Minerals, Metals and Materials Society) p 371

[29] Loiseau A 1996 Curr. Opin. Solid State Mater. Sci. 1369

[30] Kikuchi R and Cahn J W 1979 Acta Metall. 271337

[31] Turchi P E A 1994 Principles (Intermetallic Compounds vol 1) ed Westbrook J H and R L Fleischer (New York: Wiley) p 21

[32] de Fontaine D 1994 Solid State Physics vol 47 (New York: Academic) p 33

[33] Allen S M and Cahn J W 1976 Acta Metall. 24425

[34] Yang C N 1945 J. Chem. Phys. 1366

[35] Chassagne F, Bessiere M, Calvayrac Y, Cenedese P and Lefebvre S 1989 Acta Metall. 372329

[36] Khachaturyan A G 1983 Theory of Structural Phase Transformations in Solids (New York: Wiley)

[37] Beiden S V and Vaks V G 1992 Phys. Lett. A 163209

[38] Kayser F X and Stassis C 1981 Phys. Status Solidi a 64335 